\title{
Constraints on the Martian lithosphere from gravity and topography data
}

\author{
V. Belleguic, ${ }^{1,2}$, P. Lognonné, ${ }^{1}$ and M. Wieczorek, ${ }^{1}$
}

\begin{abstract}
Localized spectral admittances of the large Martian volcanoes are modeled by assuming that surface and subsurface loads are elastically supported by the lithosphere. For this purpose, a new method for calculating gravity anomalies and lithospheric deflections which is applicable when the load density differs from that of the crust is developed. The elastic thickness, crustal thickness, load density and crustal density were exhaustively sampled in order to determine their effect on the misfit between the observed and modeled admittance function. We find that the densities of the Martian volcanoes are generally well constrained with values of $3200 \pm 100 \mathrm{~kg} \mathrm{~m}^{-3}$ which is considerably greater than those reported previously. Nevertheless, such higher densities are consistent with those of the Martian basaltic meteorites, which are believed to originate from the Tharsis and Elysium volcanic provinces. The crustal density is constrained only beneath the Elysium rise to be $3270 \pm 150 \mathrm{~kg} \mathrm{~m}^{-3}$. If this value is representative of the Northern lowlands, then Pratt compensation is likely responsible for the approximatively $6 \mathrm{~km}$ elevation difference between the Northern and Southern hemispheres. The elastic thickness associated with Martian volcanoes (when subsurface loads are ignored) are found to be the following: Elysium rise $(56 \pm 20 \mathrm{~km})$, Olympus Mons $(93 \pm 40 \mathrm{~km})$, Alba Patera $(66 \pm 20$ $\mathrm{km})$, and Ascraeus Mons $(105 \pm 40 \mathrm{~km})$. We have also investigated the possible presence of subsurface loads, allowing the bottom load to be either located in the crust as dense intrusive material, or in the mantle as less dense material. We found that all volcanoes except Pavonis are better modeled with the presence of less dense material in the upper mantle, which is either indicative of a mantle plume or a depleted mantle composition. An active plume beneath the major volcanoes is consistent with recent analyses of cratering statistics on Olympus Mons and the Elysium rise, which indicate that some lava flows are as young as 10-30 Myr, as well as with the crystallization age of the Shergottites, which can be as young as 180 Myr.
\end{abstract}

\section{Introduction}

Before seismometers are deployed on Mars [e.g., Lognonné et al., 2000], gravity and topography measurements are the primary data sets from which the properties of a planet's crust and upper mantle can be constrained. A common approach is to consider that, over geological time, the lithosphere behaves as an elastic plate overlaying a fluid mantle and to model the lithospheric deformations produced by topographic and/or internal density anomalies [e.g. Turcotte et al., 1981; Forsyth, 1985]. The resulting gravity anomalies depend upon physical parameters such as the crustal and load densities and the thicknesses of the elastic lithosphere and crust.

To date, only a few localized admittance studies have been applied to Mars using recent Mars Global Surveyor (MGS) data. Using line of sight gravitational acceleration profiles, McKenzie et al. [2002] inverted for the crustal density and elastic thickness for various regions by modeling 1-D Cartesian gravitational admittances. Only surface loads were considered in that study, but this assumption was not justified by comparing observed and modeled coherence functions. It was further assumed that the load

\footnotetext{
${ }^{1}$ Département de Géophysique spatiale et planétaire, UMR7096, Institut de Physique du Globe de Paris, France

${ }^{2}$ Joint Planetary Interior Physics Research Group of the University Mnster and DLR, Berlin
}

Copyright 2005 by the American Geophysical Union. 0148-0227/05/\$9.00 density was the same as the crustal density, which is unlikely to be true for the Martian volcanoes [e.g. Neumann et al., 2004]. Using a similar approach, Nimmo [2002] modeled the mean crustal thickness, the elastic thickness and the surface density for a region centered on the hemispheric dichotomy. The theoretical admittance model utilized in his study assumed that surface and subsurface loads were uncorrelated, but this hypothesis was not tested by comparing the observed coherences with the predictions of this model. Moreover, as emphasized by Pérez-Gussinyé [2004], the results of these two studies might be biased as the employed multitaper spectral estimation procedure was not applied similarly to the data as to the theoretical model. Finally, McGovern et al. [2002] calculated admittance and coherence spectra for several volcanic regions employing the spatiospectral localization method of Simons et al. [1997]. Their models of lithospheric flexure took into account both surface and subsurface loads (assumed to be in phase) and a load density that differed from the crustal density. While $T_{e}$ and $\rho_{l}$ were constrained for certain regions, several factors have led us to reconsider their results. In particular, the degree- 1 topography was treated as a load in their study, and the surface load was not calculated in a self-consistent manner when the load density differed from the crustal density. We have further found that the modeled and observed admittances were mistakenly calculated at two different radii causing their modeled densities to be underestimated by approximately 300 $\mathrm{kg} \mathrm{m}^{-3}$ (see the correction of McGovern et al. [2004]).

In the above-mentioned studies, and in previous global ones such as Banerdt [1986] or Turcotte et al. [1981], various approximations have been made in order to calculate quickly the lithospheric deflection and the predicted gravity 
field. However, some of the employed assumptions may not be entirely applicable to Mars as it is a small planet with extreme topographic variations. As an example, the use of the "mass-sheet" approximation when computing the gravity anomaly due to a large volcano on Mars can introduce an error of up to 500 mGals, corresponding to $\sim 25 \%$ of the signal [McGovern et al., 2002]. In addition, the magnitude of this lithospheric load depends upon the local gravitational potential within the crust, and this is a function of the lithospheric deflection. While a computationally efficient method that considers finite amplitude relief in the calculation of gravity anomalies does exist in the spherical domain [Wieczorek and Phillips, 1998], this method can only calculate the potential on a reference sphere whose radius is greater than the planet's maximum radius or smaller than the minimum depth of lateral density variations, and hence is not suitable for calculating the potential at arbitrary points within a planet.

In order to improve the accuracy of the theoretical flexure and admittance models, we have here developed a new numerical method that calculates precisely both the load acting upon the lithosphere due to an arbitrary density distribution and the corresponding lithospheric deflection that is affordable from a computational point of view. As recent studies have highlighted the evidence for recent volcanic activity on Mars [Nyquist et al., 2001; Hartmann and Neukum, 2001], the martian mantle must be still dynamic, and variations in either temperature or composition are likely to play an important role in the planet's observed topography and gravity field. We have therefore investigated the possible presence of subsurface loads acting on the lithosphere, either as dense intrusive materials in the crust or less dense materials in the mantle. This later possibility could be a result of temperature anomalies in a mantle plume and/or a depleted mantle composition.

We have generated surface and subsurface loading models by exhaustively sampling all possible values (within limits) of the load density, the crustal density, the elastic and the crustal thickness and the ratio of surface to subsurface loads. The observed and modeled admittance and coherence functions between gravity and topography were then compared as a function of these model parameters for the major Martian volcanoes. In this study, we use the spectral localization procedure developed by Wieczorek and Simons [2004] and use localizing windows that concentrate $\sim 99 \%$ of their energy within the region of interest, in comparison to $\sim 93 \%$ as is the case of the windows employed by McGovern et al. [2002] (which is based on the methodology of Simons et al. [1997]).

\section{Method}

The lithospheric model used here is shown in Figure 1. It consists of a surface load, such as a volcano, with a density $\rho_{l}$ supported by a thin elastic lithosphere of thickness $T_{e}$ overlying a weak mantle that behaves as a fluid over geological time. The load deforms the lithosphere with a displacement $w$ (measured positive downward) which is presumed to be constant for each density interface. This deflection depends on several parameters, and the following are investigated in this study: the load density $\rho_{l}$, the crustal density $\rho_{c}$, the crustal thickness $T_{c}$, the elastic thickness $T_{e}$, and possible subsurface loads. The model is subdivided by five major density interfaces (see Table 1): (1) $R_{0}$, a spherical interface exterior to the planet of mean planetary radius $R,(2)$ the surface, $R+h,(3)$ the base of the crust, $R-T_{c}-w$, (4) the base of the lithosphere, $R-T_{e}-w$ and (5) a spherical interface within the non-lithospheric part of the mantle, $R-T_{c}-M$.
In this section we describe the method developed in order to compute the gravity field associated with this lithospheric deformation. The major improvement over previous studies is a more rigorous methodology in computing both the gravity anomaly and the magnitude of the load acting on the lithosphere, especially when the load density differs from that of the crustal density.

\subsection{Modeling the gravity anomaly}

Our method allows for the calculation of the gravitational potential both inside and outside of an aspherical and laterally heterogeneous planet. All calculations are performed on a grid in the space domain where the grid spacing was chosen to facilitate spherical harmonic transforms and their corresponding spatial reconstructions [e.g. Lognonné and Romanowicz, 1990; Driscoll and Healy, 1994; Sneeuw, 1994]. For an arbitrary density structure, we need to solve the gravitational differential equations

$$
\begin{aligned}
\mathbf{G} & =\nabla U, \\
\nabla \cdot \mathbf{G} & =-4 \pi \mathcal{G} \rho,
\end{aligned}
$$

both within and exterior to the planet, where $\mathbf{G}$ is the gravitational field vector (assumed to be positive upward), $U$ is the gravitational potential, $\rho$ the density and $\mathcal{G}$ the gravitational constant. G, $U$ and $\rho$ implicitly depend on the spherical coordinates $r, \theta$ and $\phi$. This set of first-order differential equations can be solved numerically if the interior density structure of the planet is known. We first compute exactly $\mathbf{G}, U$ and their first derivatives at a radius $R_{0}$ exterior to the planet using a generalization of the method described in Wieczorek and Phillips [1998], here modified to allow for lateral variations of density (see Appendix B for the details). Using the first radial derivatives (as described below) $U$ and $\mathbf{G}$ can then be estimated at a different radius using a first order Taylor series. By repeating this procedure, we can estimate the potential and gravity anywhere interior or exterior to a planet.

To avoid numerical errors and to simplify the calculations, a set of curvilinear coordinates $(s, \theta, \phi)$ is used which reduces irregular interfaces to spherical ones. The relationship between the two sets of coordinates is given by:

$$
r(s, \theta, \phi)=r_{i}(\theta, \phi)+\left[r_{i+1}(\theta, \phi)-r_{i}(\theta, \phi)\right] \frac{s-s_{i}}{s_{i+1}-s_{i}},
$$

where $s=s_{i}$ defines the mean radius of the $i^{\text {th }}$ density interface with its associated relief $r_{i}(\theta, \phi)$, while $s=s_{i+1}$ defines the mean radius of the $(i+1)^{t h}$ interface with relief $r_{i+1}(\theta, \phi)$. In practice, $s$ takes on discret values between $s_{i}$ and $s_{i+1}$ and these values will be denoted by $s_{j}$. We next transform equations 1 and 2 to this new set of coordinates and obtain expressions for the derivatives of $U$ and $\mathbf{G}$ with respect to $s$. Here we only quote the relevant results, and refer the reader to Appendix A for further details. Between $r\left(s_{i}, \theta, \phi\right)$ and $r\left(s_{i+1}, \theta, \phi\right)$ equation 1 reduces to

$$
\begin{aligned}
& \frac{\partial U}{\partial s}=C_{1} G^{r} \\
& \text { and } \\
& G^{\alpha}=D_{\alpha} U \\
&-\left[C_{2} D_{\alpha} r_{i+1}(\theta, \phi)+C_{3} D_{\alpha} r_{i}(\theta, \phi)\right] \frac{\partial U}{\partial s}
\end{aligned}
$$

where $D_{\alpha}$ is the covariant derivative with respect to the variable $\alpha$, here equal to either $\theta$ and $\phi$ or + and - (see equations A21 and $\mathrm{C} 4$ ), and the constants $C_{1}, C_{2}$ and $C_{3}$ (which depend upon $\theta$ and $\phi$ ) are given by:

$$
C_{1}=\frac{r_{i+1}(\theta, \phi)-r_{i}(\theta, \phi)}{s_{i+1}-s_{i}}
$$




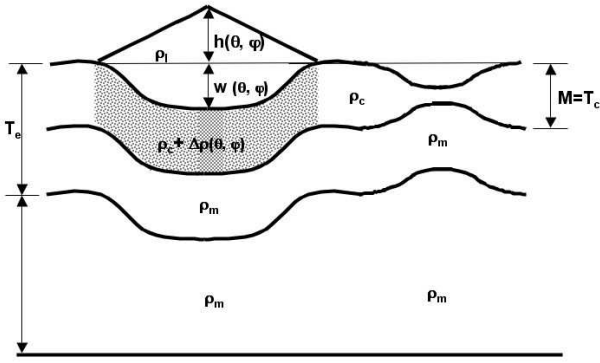

(A) $f<0$

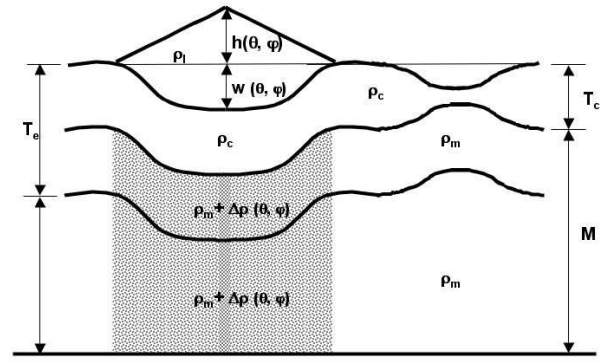

(B) $f>0$

Figure 1. Schematic representation of the lithospheric model used in the calculations. $h(\theta, \phi)$ is the surface topography, $w(\theta, \phi)$ the flexural deformation both measured with respect to the mean planetary radius. $T_{c}, T_{e}$ and $M$ are respectively the crustal thickness, the elastic thickness and the depth of the subsurface load. $\rho_{l}, \rho_{c}$ and $\rho_{m}$ are respectively the densities of the surface load, the crust and the mantle. $\Delta \rho$ is the laterally varying density anomaly, associated with subsurface loads. Two cases of buried loads are represented: one where the subsurface load is in the crust, with $M=T_{c}(\mathrm{~A})$, and the other where the subsurface load is present in the mantle to a depth $M$ (B).

$$
\begin{aligned}
C_{2} & =\frac{s-s_{i}}{r_{i+1}(\theta, \phi)-r_{i}(\theta, \phi)} \\
C_{3} & =\frac{s_{i+1}-s}{r_{i+1}(\theta, \phi)-r_{i}(\theta, \phi)} .
\end{aligned}
$$

Equation 2 yields

$$
\begin{aligned}
\frac{\partial G^{r}}{\partial s} & =-C_{1}\left[4 \pi \mathcal{G} \rho+\frac{2 G_{r}}{r}+\frac{1}{r} \nabla_{\Sigma} \cdot \mathbf{G}\right] \\
& +C_{1} \sum_{\alpha}\left[C_{2} D_{\alpha} r_{i+1}(\theta, \phi)+C_{3} D_{\alpha} r_{i}(\theta, \phi)\right] \frac{\partial G_{\alpha}}{\partial s}
\end{aligned}
$$

where $\nabla_{\Sigma} \cdot \mathbf{G}$ is horizontal divergence (see equation A30) and the summation is over the two tangential directions $\theta$ and $\phi$. In the above equations, $r, G^{r}, G^{\alpha}, U, \rho, C_{1}, C_{2}$ and $C_{3}$ are implicitly functions of the three mapped coordinates $s, \theta$ and $\phi$.

In order to determine the first derivative of $\mathbf{G}^{\mathbf{r}}$ with respect to $s$, it is necessary to calculate the horizontal components of the gravity field, $G^{\alpha}$, and its horizontal divergence. As both $U$ and $\mathbf{G}$ are initially known on a grid of $(\theta, \phi)$ for a given value of $s$, these calculations are most easily performed in the spectral domain. While the derivatives of $U$ and the surface gradient of $\mathbf{G}$ could be performed using ordinary spherical harmonics, the obtained relationships are cumbersome. These calculations are greatly facilitated by instead using the generalized spherical harmonics, as described in detail in Phinney and Burridge [1973]. In this basis, the potential and horizontal gravity components can be expressed as

$$
\begin{aligned}
U(s, \theta, \phi) & =\sum_{\ell=0}^{\mathrm{Lmax}} \sum_{m=-\ell}^{\ell} U_{\ell m}(s) Y_{\ell}^{m}(\theta, \phi), \\
G^{\alpha}(s, \theta, \phi) & =\sum_{\ell=0}^{\mathrm{Lmax}} \sum_{m=-\ell}^{\ell} G_{\ell m}^{\alpha}(s) Y_{\ell}^{\alpha m}(\theta, \phi),
\end{aligned}
$$

where $u_{\ell m}$ and $G_{\ell m}^{\alpha}$ are the complex coefficients corresponding to the generalized spherical harmonics $Y_{\ell}^{\alpha m}$, with $\alpha$ defined to be either - or + . We note that in our case, where the gravity field is real and has only a poloidal component, $G_{\ell m}^{+}=G_{\ell m}^{-}=G_{\ell m}$ [see Phinney and Burridge, 1973]. Given the coefficients $U_{\ell m}$ and $G_{\ell m}$, the horizontal derivatives of $U$ and $r(\theta, \phi)$ can be calculated using equation $\mathrm{C} 4$, and the gradient of $\mathbf{G}$ can be calculated by simple multiplications using equation C6. We note that equations 4-9 are valid using either the spherical or canonical basis. These spherical harmonics operations were performed using the software package of Clévédé and Lognonné [2002].

Once $\partial U / \partial s$ and $\partial G^{r} / \partial s$ have been obtained at a given $(s, \theta, \phi)$, we estimate $U$ and $G^{r}$ at the position $(s+\Delta s, \theta, \phi)$ using a first order Taylor series (we note that since we perform a downward propagation, $\Delta s$ is negative)

$$
\begin{aligned}
U(s+\Delta s, \theta, \phi) & =U(s, \theta, \phi)+\Delta s \frac{d U(s, \theta, \phi)}{d s}, \\
G^{r}(s+\Delta s, \theta, \phi) & =G^{r}(s, \theta, \phi)+\Delta s \frac{d G(s, \theta, \phi)}{d s} .
\end{aligned}
$$

The error associated with this approximation decreases with decreasing $\Delta s$. Based on a comparison with a more robust Runge-Kutta integration technique, we have chosen to use 50 equally spaced layers exterior to the planet where the density is zero and 100 equally spaced layers within both the crust and the mantle lithosphere.

\subsubsection{Resolution by a perturbation approach}

In order to reduce numerical errors, we have separated the gravity and the potential fields into two components: a reference component that corresponds to the case of a spherical model, and a "perturbed" component defined as the difference between the total and the reference fields. For each value of $s$, we write these fields as,

$\begin{aligned} U & =U_{0}+U_{1} \\ \mathbf{G} & =\mathbf{G}_{0}+\mathbf{G}_{1}\end{aligned}$

where the subscript 0 and 1 corresponds to the reference and perturbed terms, respectively. The reference components $U_{0}(s)$ and $G_{0}(s)$ at a radius $s$ are calculated analytically using

$$
\begin{aligned}
U_{0}(s) & =\frac{\mathcal{G}}{s}\left[M-4 \pi \int_{s}^{R} \rho_{0}(s) s^{2} d s\right] \\
G_{0}(s) & =\frac{U_{0}(s)}{s},
\end{aligned}
$$

where $M$ is the total planetary mass and $\rho_{0}$ is the mean density for a given radius $s$. We note that $r$ and $s$ are equivalent when there are no lateral variations. Since these components are solutions of equations 1 and 2, we obtain the following 
Table 1. Summary of the interfaces and densities used in the gravity field computations.

\begin{tabular}{|c|c|c|c|}
\hline \multirow{2}{*}{$\begin{array}{l}\text { Aedium } \\
\text { Aacttum }\end{array}$} & Topographic interfaces & Spherical interfaces & \multirow{2}{*}{$p_{0}$ ensities $\mathrm{m}^{-3}$} \\
\hline & $\begin{array}{l}r_{5}(\theta, \phi)=R_{0} \\
r_{4}(\theta, \phi)=R+h(\theta, \phi)\end{array}$ & $\begin{array}{l}s_{5}=R_{0} \\
s_{4}=R\end{array}$ & \\
\hline Crust & $r_{4}(\theta, \phi)=R+h(\theta, \phi)$ & $s_{4}=R$ & $\begin{array}{l}\rho_{0}= \begin{cases}\rho_{l} & \text { if } r \geq R-w(\theta, \phi) \\
\rho_{c} & \text { otherwise }\end{cases} \\
\Delta \rho(\theta, \phi)=-\frac{\rho_{l} h_{l}}{M} f \\
\quad \text { if } r_{3}(\theta, \phi) \leq r \leq R-w(\theta, \phi) \\
\quad \text { and } f<0\end{array}$ \\
\hline \multirow[t]{2}{*}{$\begin{array}{l}\text { mantle } \\
\text { Lithospheric } \\
\text { Mantle } \\
\end{array}$} & $\begin{array}{l}r_{3}(\theta, \phi)=R-T_{c}-w(\theta, \phi) \\
r_{2}(\theta, \phi)=R-T_{e}-w(\theta, \phi)\end{array}$ & $\begin{array}{l}s_{3}=R-T_{c} \\
s_{2}=R-T_{e}\end{array}$ & $\begin{array}{l}\rho_{0}=\rho_{m} \\
\Delta \rho(\theta, \phi)= \begin{cases}-\frac{\rho_{l} h_{l}}{M} f & \text { if } f>0 \\
0 & \text { if } f \leq 0\end{cases} \\
\rho_{0}=\rho_{m}\end{array}$ \\
\hline & $\begin{array}{l}r_{2}(\theta, \phi)=R-T_{e}-w(\theta, \phi) \\
r_{1}(\theta, \phi)=R-T_{c}-M\end{array}$ & $\begin{array}{l}s_{2}=R-T_{e} \\
s_{1}=R-T_{c}-M\end{array}$ & \\
\hline
\end{tabular}

relations for their radial derivatives,

$$
\begin{aligned}
\frac{\partial U_{0}}{\partial s} & =G_{0}^{r}, \\
\frac{\partial G_{0}^{r}}{\partial s} & =-4 \pi \mathcal{G} \rho_{0}-\frac{2 G_{0}^{r}}{s} .
\end{aligned}
$$

Using equations $14,15,18$ and 19 , the system of equations 4 through 9 can then be rewritten as a function of the perturbed components $U_{1}$ and $G_{1}^{r}$, as

$$
\begin{aligned}
\frac{\partial U_{1}}{\partial s} & =C_{1} G_{1}^{r}+\left(C_{1}-1\right) G_{0}^{r} \\
\frac{\partial G_{1}^{r}}{\partial s} & =-C_{1}\left[4 \pi \mathcal{G} \delta \rho(s, \theta, \phi)+\frac{1}{r} \nabla_{\Sigma} \cdot \mathbf{G}^{1}+\frac{2 G_{1}^{r}}{r}\right] \\
& +C_{1} \sum_{\alpha}\left[C_{2} D_{\alpha} r_{i+1}(\theta, \phi)+C_{3} D_{\alpha} r_{i}(\theta, \phi)\right] \frac{\partial G_{1}^{\alpha}}{\partial s} \\
& -4 \pi \mathcal{G} \rho_{0}\left(C_{1}-1\right) \\
& +\left[\frac{r_{i}(\theta, \phi) s_{i+1}-r_{i+1}(\theta, \phi) s_{i}}{s_{i+1}-s_{i}}\right]\left(\frac{2 G_{0}^{r}}{s r}\right)
\end{aligned}
$$

where $\delta \rho(s, \theta, \phi)=\rho(s, \theta, \phi)-\rho_{0}$ is the lateral variation in density. Equation 5 gives us the supplementary equation

$$
\begin{aligned}
G_{1}^{\alpha} & =D_{\alpha} U_{1} \\
& -\left[C_{2} D_{\alpha} r_{i+1}(\theta, \phi)+C_{3} D_{\alpha} r_{i}(\theta, \phi)\right]\left(\frac{\partial U_{1}}{\partial s}+\frac{\partial U_{0}}{\partial s}\right) .
\end{aligned}
$$

In the above equations $\rho_{0}$ is a function of $s$ and as before $U_{1}, G_{1}^{r}$ and $G_{1}^{\alpha}$ depend upon $s, \theta$ and $\phi$.

\subsubsection{Summary: A recipe for computing the gravity} field

A series of equations have been presented is this section and we recall here for clarity the main steps involved in computing the gravity field anywhere within or exterior to body possessing an arbitrary density structure.

1. The first step is to define a grid in the space domain for which $U$ and $\mathbf{G}$ will be computed. It is convenient to used a grid that is equally spaced in the $\phi$ direction, and irregularly spaced in the $\theta$ direction according to the zeros of the Legendre polynomial $P_{2 \mathrm{~L}_{\max }-1}$. With such a grid, the spherical harmonics transform used to compute the horizontal derivatives and gradients is easily performed [see Lognonné and Romanowicz, 1990; Driscoll and Healy, 1994; Sneeuw, 1994]. The grid used here is $128 \times 256$.

2. Next, $U(s, \theta, \phi)$ and $G^{r}(s, \theta, \phi)$ are computed on this grid at a radius $s=R_{0}$ above the mean planetary radius using the method described in Appendix B. $U_{1}\left(R_{0}, \theta, \phi\right)$ and $G_{1}^{r}\left(R_{0}, \theta, \phi\right)$ are then obtained by subtracting the reference field $U_{0}\left(R_{0}, \theta, \phi\right)$ and $G_{0}^{r}\left(R_{0}, \theta, \phi\right)$ as defined by equations 16 and 17. $G_{1}^{\alpha}\left(R_{0}, \theta, \phi\right)=D_{\alpha} U^{1}\left(R_{0}, \theta, \phi\right)$ is then calculated using equation $\mathrm{C} 4$ after expressing $U_{1}\left(R_{0}, \theta, \phi\right)$ in term of generalized spherical harmonics according to equation $\mathrm{C} 2$.

3. In order to initiate the propagation of the fields, the main density interfaces have to be defined (here we defined these as the surface, the base of the crust and the base of the lithosphere). For each layer between $r_{i}(\theta, \phi)$ and $r_{i+1}(\theta, \phi)$, the angular derivatives $D_{\alpha} r_{i}$ and $D_{\alpha} r_{i+1}$, which are required in equations 21 and 22 , are computed at all grid points, using equation $\mathrm{C} 4$.

4. On each sublayer $s_{j}$ between $s_{i}$ and $s_{i+1}, U_{0}$ and $G_{0}^{r}$ are computed at all grid points using equations 16 and 17 , and $r(s, \theta, \phi)$ is calculated using equation $3 . D_{\alpha} U^{1}\left(s_{j}, \theta, \phi\right)$ is then calculated using equation $\mathrm{C} 4$ and with this quantity $\partial U_{1} / \partial s$ is computed using equation 20 .

5. $G_{1}^{\alpha}$ is then calculated at all grid points using equation 22 , and $\partial G_{1}^{\alpha} / \partial s$ is estimated using a finite difference formula between two layers:

$$
\frac{\partial G_{1}^{\alpha}\left(s_{j}, \theta, \phi\right)}{\partial s}=\frac{G_{1}^{\alpha}\left(s_{j}-\Delta s, \theta, \phi\right)-G_{1}^{\alpha}\left(s_{j}, \theta, \phi\right)}{\Delta s} .
$$

6. Expressing $G_{1}^{\alpha}$ in spherical harmonics, $\nabla_{\Sigma} \cdot \mathbf{G}_{1}$ can be computed using equation C6. $\partial G_{1}^{r} / \partial s$ can then similarly be computed using equation 21.

7. Finally, $U_{1}\left(s_{j}+\Delta s, \theta, \phi\right)$ and $G_{1}^{r}\left(s_{j}+\Delta s, \theta, \phi\right)$ are obtained using $U_{1}\left(s_{j}, \theta, \phi\right), G_{1}^{r}\left(s_{j}, \theta, \phi\right), \partial U^{1} / \partial s$ and $\partial G_{1}^{r} / \partial s$ in equations 12 and 13 .

8. The steps 4-7 are continued for all values of $s_{j}$ between $s_{i}$ and $s_{i+1}$ and when the next major interface is reached, one restarts at step 3 .

\subsection{Modeling the lithospheric deflection}

Assuming that the rheology of planetary bodies can be approximated by that of a fluid mantle overlying an elastic lithosphere, the relationship that links the pressure acting on the shell $p$ to the vertical displacement $w$ is [Kraus, 1967; Turcotte et al., 1981]

$$
\begin{aligned}
D \nabla^{6} w+4 D \nabla^{4} w+E T_{e} R^{2} \nabla^{2} w+ & 2 E T_{e} R^{2} w= \\
& R^{4}\left(\nabla^{2}+1-\nu\right) p
\end{aligned}
$$

where $D=E T_{e}^{3} / 12\left(1-\nu^{2}\right)$ is the flexural rigidity, $E$ is Young's modulus, $\nu$ is Poisson's ratio, $T_{e}$ is the elastic thickness and $R$ is the radius of the shell, here taken to be the mean planetary radius. The pressure $p$ and the displacement $w$ (measured positive downward) depends upon position $\theta$ and $\phi$ (we note that Turcotte et al. [1981] omit a $4 D \nabla^{2} w$ in equation 24 [Willemann and Turcotte, 1981] when is in practice negligible. In this equation, it is implicitly assumed that the thickness of the lithosphere is everywhere the same and small with respect to the planetary radius [see Zhong and 
Zuber, 2000]. Given that the expected elastic thicknesses are much less than a tenth of the planetary radius, this thin shell approximation should not gives rise to any appreciable errors. In addition, horizontal forces caused by topography along density interfaces are neglected, as in most previous studies [e.g., Kraus, 1967; Turcotte et al., 1981; McGovern et al., 2002], since they only have a small influence on the vertical displacement [see Banerdt, 1986].

The net load $p$ on the lithosphere is the difference between the gravitational force per unit area acting on the lithosphere, $q_{a}$, and the hydrostatic pressure within the fluid mantle $q_{h}$ at the base of the lithosphere:

$$
p(\theta, \phi)=q_{a}(\theta, \phi)-q_{h}(\theta, \phi) .
$$

In previous studies, this load has been expressed in a first order form in order to obtain a linear relation between the surface topography $h$, and the lithospheric deflection $w$. For example, Turcotte et al. [1981] employed a linear relationship between topography and gravity, and assumed that the geoid and gravitational acceleration did not vary with depth in the elastic shell, giving rise to the equation

$$
p=g\left[\rho_{c} h-\rho_{m} h_{g}-\left(\rho_{m}-\rho_{c}\right) w\right],
$$

where $\rho_{c}$ and $\rho_{m}$ are, respectively, the density of the crust and mantle and $h_{g}$ is the geoid (i.e., the height to an equipotential surface). Other methods have been employed which yield similar linear relationship between $p$ and $w$. In Banerdt [1986], the geoid was not assumed to be constant at all depths in the lithosphere, and both the geoid at the surface and at the base of the crust-mantle interface (i.e., the Martian "Moho") appear in the expression of $p$ (see Appendix D). In McGovern et al. [2002], $p$ is defined in a similar manner to Banerdt [1986] except that the topography was referenced to the observed geoid and the geoid was assumed to be zero at the base of the crust. In this latter study, the load density, $\rho_{l}$, was allowed to differ from the crustal density, but as we show in Appendix D (and section 3.1), the equations that were used are not entirely self consistent.

In this section, we describe a self consistent method by which the lithospheric load $p$ and the corresponding deflection $w$ are determined exactly within the framework of a thin elastic spherical shell formulation. We will see that the load $p$ depends upon the value of $U$ inside the planetary body, which is easily calculated using our method described in section 2.1 .

2.2.1. Gravitational force acting on the lithosphere

The vertical gravitational force due to the load acting on an infinitesimal volume $V$ of lithosphere centered at $\theta, \phi$ is:

$$
F=d \Omega \int_{R-T_{e}-w}^{R+h} G_{r}(r, \theta, \phi) \rho(r, \theta, \phi) r^{2} d r,
$$

where $G_{r}$ is the vertical component of the gravity field (defined positive upward), $R$ is the mean planetary radius, and $d \Omega$ is the associated differential solid angle. The gravitational force per unit surface area is then given by

$$
q_{a}=\frac{F}{d \Omega R^{2}} .
$$

Since $G_{r}=\frac{\partial U}{\partial r}$ we deduce that

$q_{a}=\frac{1}{R^{2}} \int_{R-T_{e}-w}^{R+h} \frac{d U}{d r} \rho(r, \theta, \phi) r^{2} d r=\frac{1}{R^{2}} \int_{U\left(R-T_{e}-w\right)}^{U(R+h)} \rho(r, \theta, \phi)$

To avoid the usual approximation $r^{2}=R^{2}, q_{a}$ is calculated numerically during the propagation of $U$ from the surface to the base of the lithosphere by

$$
\left.q_{a}=\frac{1}{R^{2}} \sum_{j=1}^{N} \rho(r, \theta, \phi) r^{2}\left(s_{j}, \theta, \phi\right)\left[U_{j+1}(\theta, \phi)-U_{j}(\theta, \phi)\right] 28\right)
$$

where $N$ is the number of layers in the lithosphere.

2.2.2. Hydrostatic pressure acting on the base of the lithosphere

The equation of hydrostatic equilibrium gives

$$
\frac{d q_{h}}{d r}=\rho(r) G_{r}(r, \theta, \phi)=\rho(r) \frac{d U(r, \theta, \phi)}{d r},
$$

where we have assumed that the density of the fluid mantle is constant. Integrating the above equation yields

$$
\int_{R-T_{e}-w}^{R_{r e f}} d q_{h}=\rho_{m} \int_{R-T_{e}-w}^{R_{\text {ref }}} d U
$$

and if the reference surface is chosen as having a constant pressure and potential at the origin, then this can be rewritten as

$$
q_{h}\left(R-T_{e}-w\right)=\rho_{m} U\left(R-T_{e}-w\right)-\rho_{m} U(0)+q_{h}
$$

Since $q_{h}(0)$ and $U(0)$ are by definition constant values, they do not give rise to lateral variations in lithospheric deflection when the load is decomposed in the spectral domain. In the case where the effective elastic thickness is smaller than the crustal thickness $T_{c}$, the hydrostatic pressure produced by the fluid mantle is here presumed to act directly on the base of the crust. In this case, the pressure is given by the expression

$$
q_{h}\left(R-T_{c}-w\right)=\rho_{m} U\left(R-T_{c}-w\right)+\text { constant }
$$

The total load $p$ acting on the lithosphere can finally be expressed as

$$
\begin{aligned}
p(\theta, \phi) & =\frac{1}{R^{2}} \sum_{j=1}^{N} \rho(r, \theta, \phi) r^{2}\left(s_{j}, \theta, \phi\right)\left[U_{j+1}(\theta, \phi)-U_{j}(\theta, \phi)\right] \\
& -\left\{\begin{array}{l}
\rho_{m} U\left(R-T_{e}-w, \theta, \phi\right)+\mathrm{constant} \text { if } T_{e}>T_{6} \\
\left.\rho_{m} U\left(R-T_{c}-w, \theta, \phi\right)+\mathrm{constant} \text { if } T_{e} \leq \mathcal{T}_{c}\right)
\end{array}\right.
\end{aligned}
$$

where $N$ corresponds to the number of layers used within the lithosphere.

2.2.3. Determination of the lithospheric deflection

Following Turcotte et al. [1981], we define the dimensionless parameters

$$
\tau=\frac{E T_{e}}{R^{2}}
$$

and

$$
\sigma=\frac{D}{R^{4}}=\frac{\tau}{12\left(1-\nu^{2}\right)}\left(\frac{T_{e}}{R}\right)^{2} .
$$

Using the relationship $\nabla^{2} Y_{\ell}^{m}=-\ell(\ell+1) Y_{\ell}^{m}$, equation 24 can then be expressed in the spectral domain as

$$
\begin{aligned}
\sigma\left[\ell^{3}(\ell+1)^{3}-4 \ell^{2}(\ell+1)^{2}\right]+ & \tau[\ell(\ell+1)-2] w_{\ell m}= \\
& {[\ell(\ell+1)-(1-\nu)] p_{\ell m} }
\end{aligned}
$$

where $p_{\ell m}$ and $w_{\ell m}$ are the spherical harmonics coefficients of $p$ and $w$. Since $p$ explicitly depends upon $w$, we solve eqdations 32 and 33 in an iterative manner. First, we assume that there is initially no deflection and then calculate the load by equation 32 and the corresponding deflection by equation 33. Next, using this approximation of the deflection, we again calculate the load and deflection by equations 
32 and 33. We continue with this iterative procedure until the deflection has converged to a stable value.

\subsubsection{Treatment of the first two degrees of the to-} pography

Special care must be taken with the degree 1 and 2 terms, as these may have a different origin than our presumed load model. The degree- 1 topography is assumed here to be isostatically supported and thus, the corresponding degree- 1 Moho relief was chosen such that the degree- 1 gravity field is zero (as is required to be in center of mass coordinates). In contrast, McGovern et al. [2002] treated this term as a load, and thus the higher topography of the southern highlands was assumed to have a density equal to that of the load.

The $J_{2}$ term of the gravity and topography contains a large component directly related to the hydrostatic flattening of the planet. When computing our flexure models, only $5 \%$ of the topographic $J_{2}$ term was assumed to be due to the load [e.g. Zuber and Smith, 1997] and after the deflections were determined, the remaining $95 \%$ of the relief was added to all density interfaces. We note that the difference in removing $90 \%$ or $95 \%$ or $99 \%$ of this term has little influence on the results presented here.

\subsection{Modeling of the subsurface load}

We parameterize the subsurface load to be proportional to the surface load by a factor $f$ and consider two cases which are determined by its sign: either the load is a positive density anomaly in the crust (such as a magmatic intrusion) or a density deficit in the mantle (such as a plume or depleted mantle composition). By definition, the two loads are in phase, and the ratio $f$ is defined by

$$
f=\frac{\Delta \rho M}{\rho_{l} h_{l}},
$$

where $\mathrm{M}$ and $\Delta \rho$ are the thickness and density anomaly of the subsurface load, $h_{l}=h-w$ and $\rho_{l}$ are the thickness and density of the surface load (see the schematic representation in Figure 1). If $f<0$, a positive density perturbation is

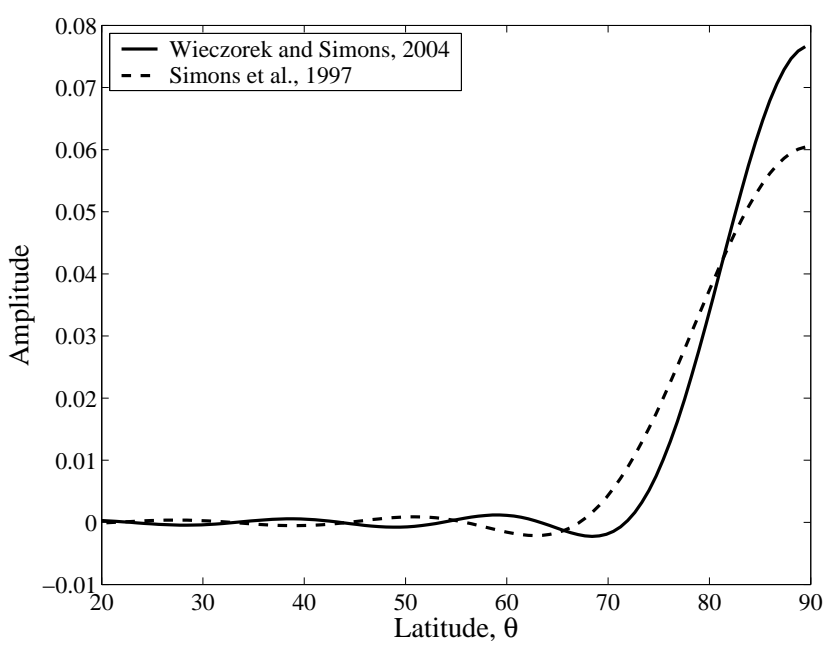

Figure 2. Spatial representation of the bandlimited window of Wieczorek and Simons [2004] (solid line) and the spectrally truncated spherical cap window of Simons et al. [1997] (dashed line) for a spatial diameter $\theta_{o}=15^{\circ}$. For the first, $L_{w i n}=16$, and the concentration factor is $98.8 \%$. The second corresponds to $L_{w i n}=15$, and possesses a concentration factor of $93.1 \%$. located exclusively in the crust with a thickness $M=T_{c}$. In contrast, if $f>0$, then a negative density perturbation is located in the mantle. For this case, the value of the subsurface load thickness was set to a single value, $M=250$ $\mathrm{km}$, as exploring all possible values of $M$ would be too time consuming from a computational point of view. This value approximately corresponds to the expected depth of melting within a plume beneath the Tharsis province [e.g., Kiefer, 2003].

\subsection{Admittance localization}

Localized admittances and coherences were calculated by windowing the gravity signal and surface topography with the bandlimited localization windows of Wieczorek and Simons [2004]. The quality of spatial localization for these windows depends upon its spectral bandwidth, $L_{\text {win }}$, and we chose this value such that $\sim 99 \%$ of the window's power was concentrated within the region of interest. Figure 2 displays the shape of this window as well as the equivalent spectrally truncated spherical cap window of Simons et al. [1997] that was subsequently used in McGovern et al. [2002]; Lawrence and Phillips [2003]; Smrekar et al. [2003] and Hoogenboom et al. [2004]. The windows represented here both correspond to a spatial diameter of $\theta_{o}=15^{\circ}$ and the spatial concentration of these are $98.8 \%$ for the window of Wieczorek and Simons [2004] and $93.1 \%$ for the corresponding window used in $\mathrm{Mc}$ Govern et al. [2002].

Spectral and cross-spectral estimates of $r(\theta, \phi)$ and $g(\theta, \phi)$ are obtained by multiplying these by the window $h_{w}(\theta, \phi)$ in the space domain, and then expanding these in spherical harmonics. If $\Psi$ and $\Gamma$ are the localized fields of $r(\theta, \phi)$ and $g(\theta, \phi)$ in space domain respectively,

$\Psi=r(\theta, \phi) h_{w}(\theta, \phi)$
$\Gamma=g(\theta, \phi) h_{w}(\theta, \phi)$

then their localized cross spectral power estimate $S_{\Psi \Gamma}$ is

$$
S_{\Psi \Gamma}(\ell)=\sum_{m=-\ell}^{\ell} \Psi_{\ell m} \Gamma_{\ell m},
$$

where $\Psi_{\ell m}$ and $\Gamma_{\ell m}$ are the spherical harmonics coefficients of $\Psi$ and $\Gamma$ respectively. The admittance, the coherence and the standard deviation of the admittance are respectively defined by:

$$
\begin{gathered}
Z_{\ell}=\frac{S_{\Psi \Gamma}(\ell)}{S_{\Psi \Psi}(\ell)}, \\
\gamma_{\ell}=\frac{S_{\Psi \Gamma}(\ell)}{\sqrt{S_{\Psi \Psi}(\ell) S_{\Gamma \Gamma}(\ell)}}, \\
\sigma_{Z}^{2}(\ell)=\left(\frac{S_{\Gamma \Gamma}(\ell)}{S_{\Psi \Psi}(\ell)}\right)\left(\frac{1-\gamma_{\ell}^{2}}{2 \ell}\right) .
\end{gathered}
$$

In the last equation, it is implicitly assumed that $\Gamma_{\ell m}=$ $Z_{\ell} \Psi_{\ell m}$ and that a coherence less than 1 is characteristic of noise.

As each windowed admittance at degree $\ell$ is influenced by all degrees in the range $\left|\ell-L_{w i n}\right| \leq \ell \leq \ell+L_{\text {win }}[S i$ mons et al., 1997; Wieczorek and Simons, 2004], we have considered a restricted range of wavelengths in our misfit calculations. Firstly, since the degree-2 term is mainly associated with the rotational flattening of the planet, and the first 6 degrees are largely a result of the Tharsis rise which may possess an elastic thickness that is different than the superposed volcanoes [e.g. Zuber and Smith, 1997; Phillips et al., 2001; Wieczorek and Zuber, 2004], we only considered the degrees $\ell>L_{w i n}+6$. Secondly, as the gravity 


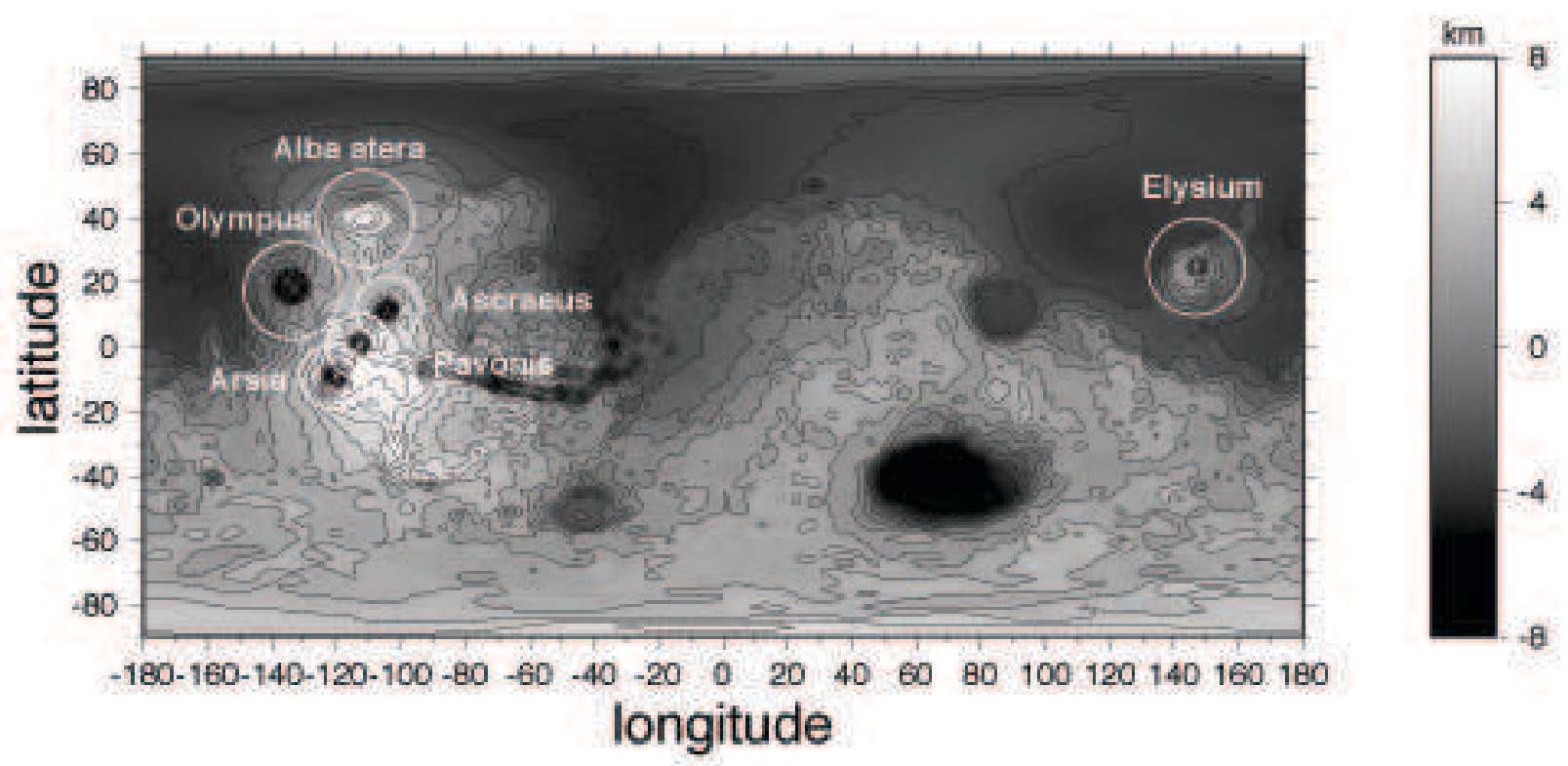

Figure 3. Position and size of the localization windows used in this study superimposed on a global topography on Mars.

Table 2. Parameter values used in the flexure model.

\begin{tabular}{lcccl}
\hline Parameter & Symbol & Value & Increment & Unit \\
\hline mean planetary radius & $R$ & 3389.5 & - & $\mathrm{km}$ \\
Young's modulus & $E$ & $10^{11}$ & - & $\mathrm{Pa}$ \\
Poisson's ratio & $\nu$ & 0.25 & - & - \\
mantle density & $\rho_{m}$ & 3500 & - & $\mathrm{kg} \mathrm{m}^{-3}$ \\
crustal density & $\rho_{c}$ & $2700-3400$ & 100 & $\mathrm{~kg} \mathrm{~m}^{-3}$ \\
load density & $\rho_{l}$ & $2700-3400$ & 100 & $\mathrm{~kg} \mathrm{~m}^{-3}$ \\
elastic thickness & $T_{e}$ & $0-200$ & 5 & $\mathrm{~km}$ \\
crustal thickness & $T_{c}$ & $30-90$ & 10 & $\mathrm{~km}$ \\
load ratio & $f$ & $-0.3-0.5$ & & \\
subsurface load thickness & $M$ & 250 & - & $\mathrm{km}$ \\
\hline
\end{tabular}
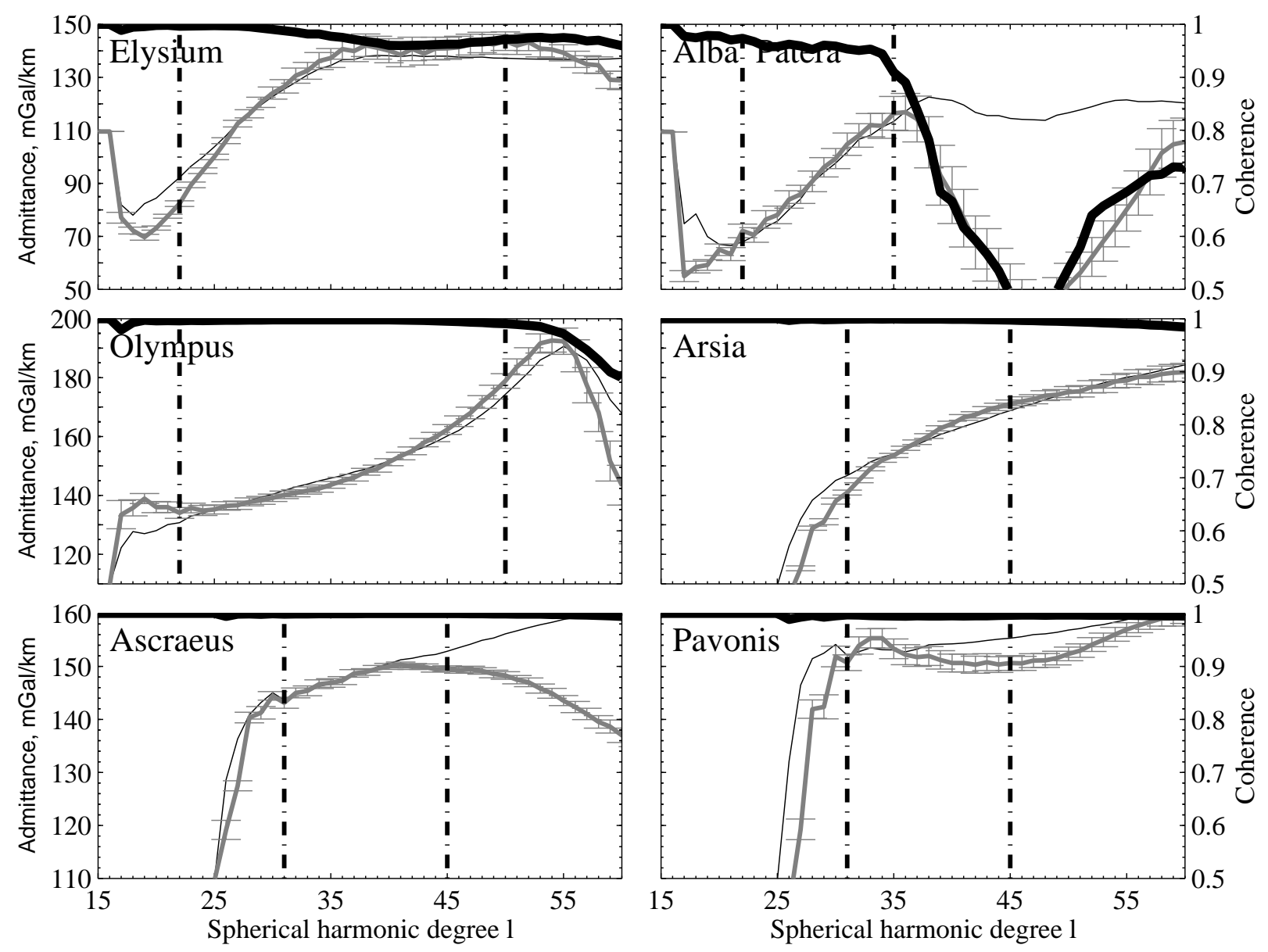
rise, we chose a window size of $15^{\circ}$, corresponding to a bandwidth of $L_{w i n}=16$. For the Tharsis Montes, which are very close to one another, we chose a value of $10^{\circ}$ corresponding to $L_{\text {win }}=25$. Figure 3 shows the locations of the volcanoes investigated here, as well as the size of the employed localization windows. Finally, we note that our loading model, in which the load and deflection are in phase, requires a coherence close to unity (the coherence is always greater than 0.99 for our forward models). This condition is satisfied for all volcanoes with the exception of Alba Patera at large $\ell$, and to a lesser extent for Elysium where the observed coherence is only 0.95 for $\ell>30$. For Alba Patera we have only considered degrees up to $\ell=35$ in our misfit calculations whereas for Elysium, we have used the entire range. Example observed admittance and coherence spectra are displayed in Figure 4.

Finally, as described below, we calculated the reduced $\chi^{2}$ and marginal probability distributions associated with the observed and theoretical admittance spectra in order to quantify the acceptable range of parameters. Table 2 summarizes all the parameter values used in this study. The assumed mantle density is $3500 \mathrm{~kg} \mathrm{~m}^{-3}$, which is consistent with the geochemical model of Sohl and Spohn [1997] and Bertka and Fei [1998].

\section{Results}

\subsection{Comparison with other methods}

The main difference between our method and that of previous studies lies in how we calculate the load acting on the lithosphere. Previous studies have used a variety of simplifying assumptions in order to calculate this, and we show in Appendix D how the load expressions of Turcotte et al. [1981], Banerdt [1986] and McGovern et al. [2002] are related to our equation 32. For comparison, Figure 5 displays the predicted gravity signal of Elysium Mons using our technique as well as that of Turcotte et al. [1981], Banerdt [1986] and McGovern et al. [2002]. We note that the equations of Appendix D are used only to compute the load and corresponding deflections, and once the deflection is computed, the same method of calculating the gravity anomaly is used. In contrast, the studies of Turcotte et al. [1981] and Banerdt [1986] used a first order mass sheet approximation when calculating the geoid and gravity signals.

The errors associated with the different approximations used in previous methods of calculating the load magnitude depends upon the values of $T_{e}, \rho_{c}$ and $\rho_{l}$. Figure 5 first shows that the methods of Turcotte et al. [1981], Banerdt [1986] and McGovern et al. [2002] give approximatively the same result when the load and crustal density are the same, and that the difference among these is greatest when the elastic thickness is small, or when the density is high. Secondly, we note that the load equations used in these previous studies are not explicitly valid when the load density differs from that of the crust (see Appendix D). In order to take this case into account, McGovern et al. [2002] have simply replaced the term $\rho_{c}$ by $\rho_{l}$ in equation D5 when computing the lithospheric load and the associated deflection. This approach, however, neglects the density contrast between the load and the crust, and uses an incorrect density contrast between the crust and the mantle. We use this approach to compute the magnitude of the load and deflection when $\rho_{l} \neq \rho_{c}$ for all previous studies even though Turcotte et al. [1981] and Banerdt [1986] never explicitly considered this case. If one models the difference between the load and crustal density as was done in McGovern et al. [2002], then it is seen that the errors can become very large (when $T_{e}=10 \mathrm{~km}, \rho_{c}=2900 \mathrm{~kg}$ $\mathrm{m}^{-3}$ and $\rho_{l}=3200 \mathrm{~kg} \mathrm{~m}^{-3}$ the error is approximately $30 \%$ ).

\subsection{Surface loading results}

In order to reduce the amount of computation time and to more fully explore the model parameter space, we first consider the case where only surface loads are present (the addition of subsurface loads will be considered in section 3.3). Theoretical gravity fields were calculated as a function of crustal density $\rho_{c}$, crustal thickness $T_{c}$, load density $\rho_{l}$, and elastic thickness $T_{e}$. In contrast to previous studies which have only investigated the misfit between the observed and modeled admittance by varying two parameters while keeping the others fixed, we have exhaustively explored this four-dimensional parameter space using the values listed in Table 2 (for this case, $f$ was set to zero). The topographic model used in this study is Mars2000.shape [Smith et al., 2001], and the employed gravity model is jgm85h02 [Lemoine et al., 2001]. While the coefficients of the gravity field are given up to degree 85 , we only interpret the associated fields up to degree 60 as the correlation between the gravity and topography dramatically decreases after this value. We then calculated the localized admittance and coherence spectra for the major Martian volcanoes: Olympus Mons, Alba Patera, the three Tharsis Montes (Arsia, Pavonis and Ascraeus) and the Elysium rise. As an example, Figure 4 shows the observed admittance and coherence as well as the best-fit admittance spectra that we have obtained for each volcano. In general, our best fit model matches the observed admittance well, indicating that the assumption of a simple surface loading model is valid for the volcanoes. The major exceptions are Arsia and Elysium, where the low angular degrees are not well modeled. We will show in section 3.3 that the misfit for these low degrees decreases with the inclusion of subsurface loads.

We quantify the permissibility of our models in two ways. First, in order to quantify the quality of fit between the observed and theoretical admittances, we calculated for each model the reduced chi squared [e.g. Press et al., 1992, chap. $15]$ :

$\frac{\chi^{2}}{\nu}\left(\rho_{c}, \rho_{l}, T_{e}, T_{c}\right)=\frac{1}{\nu} \sum_{\ell=L_{w i n}+6}^{L_{o b s}-L_{\text {win }}} \frac{\left[Z_{\ell}^{\text {obs }}-Z_{\ell}^{\text {calc }}\left(\rho_{c}, \rho_{l}, T_{e}, T_{c}\right)\right]^{2}}{\sigma_{\ell}^{2}}$

where $Z_{o b s}^{\ell}$ and $Z_{c a l c}^{\ell}$ are the observed and modeled admittances for a given degree $\ell, \sigma_{\ell}$ is the error associated with each observed admittance (see equation 40), and $\nu=L-p$ is the number of degrees of freedom, where $L$ is the number of degrees considered $\left(L=L_{o b s}-6-2 L_{w i n}+1\right)$ and $p$ is the number of model parameters (4 in this case). If the model is an accurate representation of the underlying physical process and if the measurement errors are Gaussian, then the expectation of $\frac{\chi^{2}}{\nu}$ is unity with a standard deviation given by

$$
\sigma_{\frac{\chi^{2}}{\nu}}=\sqrt{\frac{2}{\nu}}
$$

In the following figures, we have plotted the minimum value of $\frac{\chi^{2}}{\nu}\left(\rho_{c}, \rho_{l}, T_{e}, T_{c}\right)$ as a function of a single parameter. Second, we plot the marginal probability for a given parameter (the probability that a parameter $X_{i}$ possesses a value $x$ regardless of the value of the other parameters). This is given by [e.g., Tarantola, 1987]

$$
P\left(X_{i}=x\right)=C \sum_{j, k, t} \exp \left[-\frac{1}{2} m\left(X_{i}, X_{j}, X_{k}, X_{t}\right)\right]
$$

where $m\left(X_{i}, X_{j}, X_{k}, X_{t}\right)$ is the average misfit between observed and theoritical admittance spectra, defined by

$m\left(X_{i}, X_{j}, X_{k}, X_{t}\right)=\frac{1}{L} \sum_{\ell=L_{w i n}-L_{o b s}}^{L_{o b s}-L_{w i n}} \frac{\left[Z_{o b s}^{\ell}-Z_{c a l c}^{\ell}\left(\rho_{l_{i}}, \rho_{c_{j}}, T_{E_{k}}, T_{C_{t}}\right)\right]^{2}}{\sigma_{\ell}^{2}}$ 


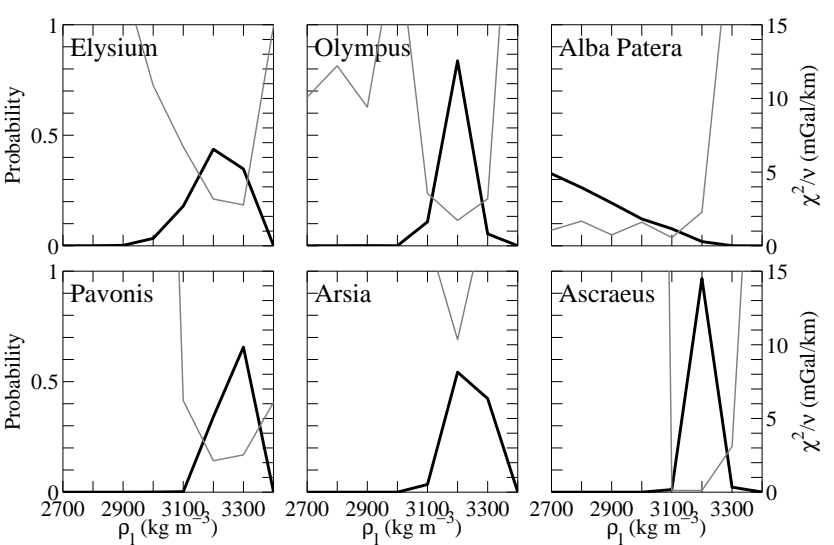

Figure 6. Left axis (black bold curve): Marginal probability as a function of $\rho_{l}$. Right axis (gray light curve): Minimum $\chi^{2} / \nu$.

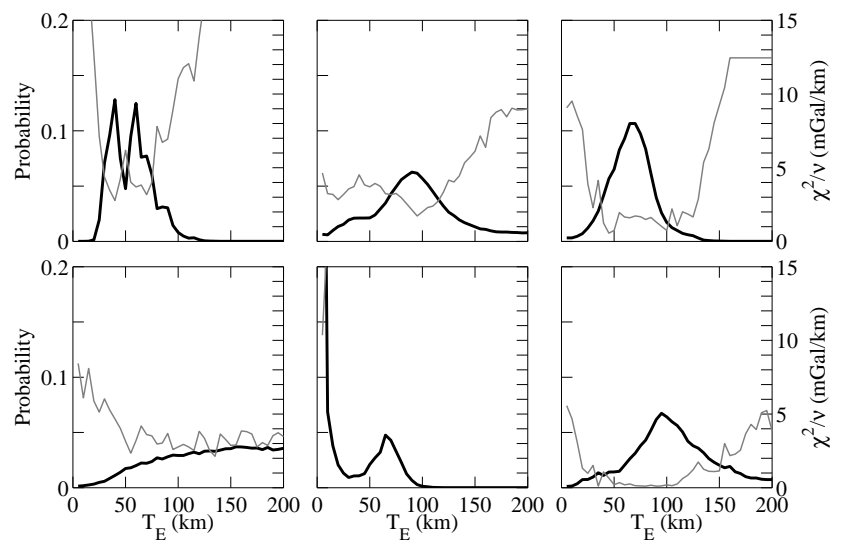

Figure 7. Left axis: Marginal probability as a function of $T_{e}$. Right axis: Minimum $\chi^{2} / \nu$.

and $\mathrm{C}$ is a constant obtained by normalizing the sum of the probabilities to 1 (we use a single average misfit as opposed to adding each misfit in quadrature because the $Z^{\ell}$ s are not linearly independent as as result of the windowing procedure). The minimum values of $\frac{\chi^{2}}{\nu}$ are mainly used to quantify the appropriateness of the model's assumptions (an acceptable model is required to have $\frac{\chi^{2}}{\nu} \sim 1$ ). In contrast marginal probabilities will be used to estimate most probable values and their associated uncertainties. When the width of the distribution can not be accurately estimated because it is smaller than the sampling interval of the parameter, we conservatively quote the uncertainty as the sampling interval.

Figures 6 to 9 display the minimum $\chi^{2} / \nu$ and the marginal probability distribution as a function of a single parameter. For all volcanoes, except Elysium and Arsia, the value of the minimum $\chi^{2} / \nu$ is close to 1 , implying that a simple elastic support model of a surface load is consistent with the data. In the case of Elysium and Arsia, the minimum $\chi^{2} / \nu$ is considerably higher $(\sim 3$ for Elysium and $\sim 9$ for Arsia). Figure 4 shows that the admittance function is a poor fit to the data at the lowest angular degrees for these two volcanoes, suggesting the need of incorporating subsurface loads in the admittance model.

\subsubsection{Constraints on the load density}

Figure 6 shows the minimum reduced $\chi^{2}$ and the marginal probability distribution as a function of load density. With the exception of Alba Patera, $\rho_{l}$ appears to be well constrained with a value of $\rho_{l}=3200 \pm 100 \mathrm{~kg} \mathrm{~m}^{-3}$. While the marginal probability distribution is not peaked for Alba Patera, lower densities are implied, and there is a $98 \%$ probability that the density is less than $3100 \mathrm{~kg} \mathrm{~m}^{-3}$. Nevertheless, we note that there are models that can fit the data to within $1-\sigma$ for any value of $\rho_{l}$ less than $3100 \mathrm{~kg} \mathrm{~m}^{-3}$.

\subsubsection{Constraints on the elastic thickness}

Figure 7 shows our results for the elastic thickness $T_{e}$. The marginal probability distributions are somewhat peaked for most of the volcanoes, implying an elastic thickness of $56 \pm 20 \mathrm{~km}$ for the Elysium rise, $93 \pm 40 \mathrm{~km}$ for Olympus Mons, $66 \pm 20 \mathrm{~km}$ for Alba Patera, and $105 \pm 40 \mathrm{~km}$ for Ascraeus Mons. For Pavonis Mons, the elastic thickness is not constrained and the marginal probability and minimum $\chi^{2} / \nu$ are nearly flat for $T_{e}>50 \mathrm{~km}$. For Arsia Mons, the probability distribution is somewhat bi-modal, with the largest mode being for near-zero elastic thicknesses. Nevertheless, we do not have much confidence in this latter result as our best-fitting model does not reproduce well the observed admittances for the lowest degrees and $\chi^{2} / \nu$ is here much greater than 1 . As will be shown in the next section, subsurface loads (such as a mantle plume) are required in

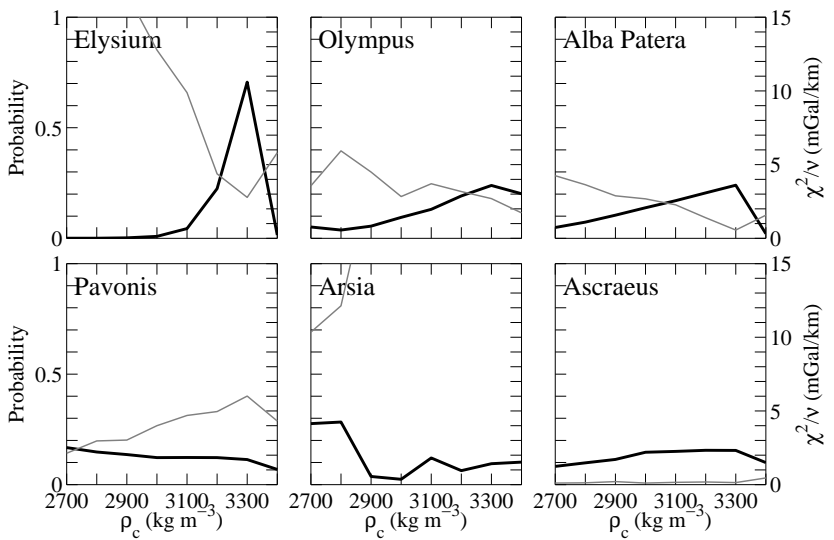

Figure 8. Left axis: Marginal probability as a function of $\rho_{c}$. Right axis: Minimum $\chi^{2} / \nu$.
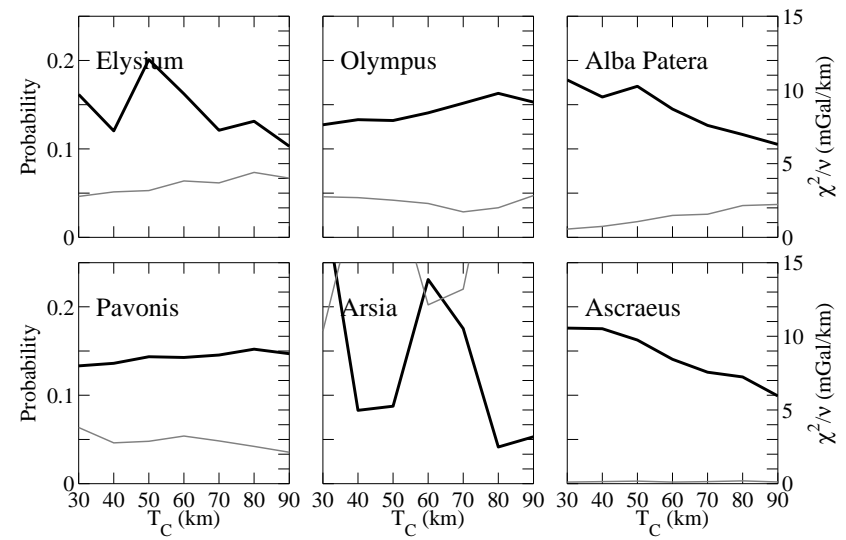

Figure 9. Left axis: Marginal probability as a function of $T_{c}$. Right axis: Minimum $\chi^{2} / \nu$. 
A
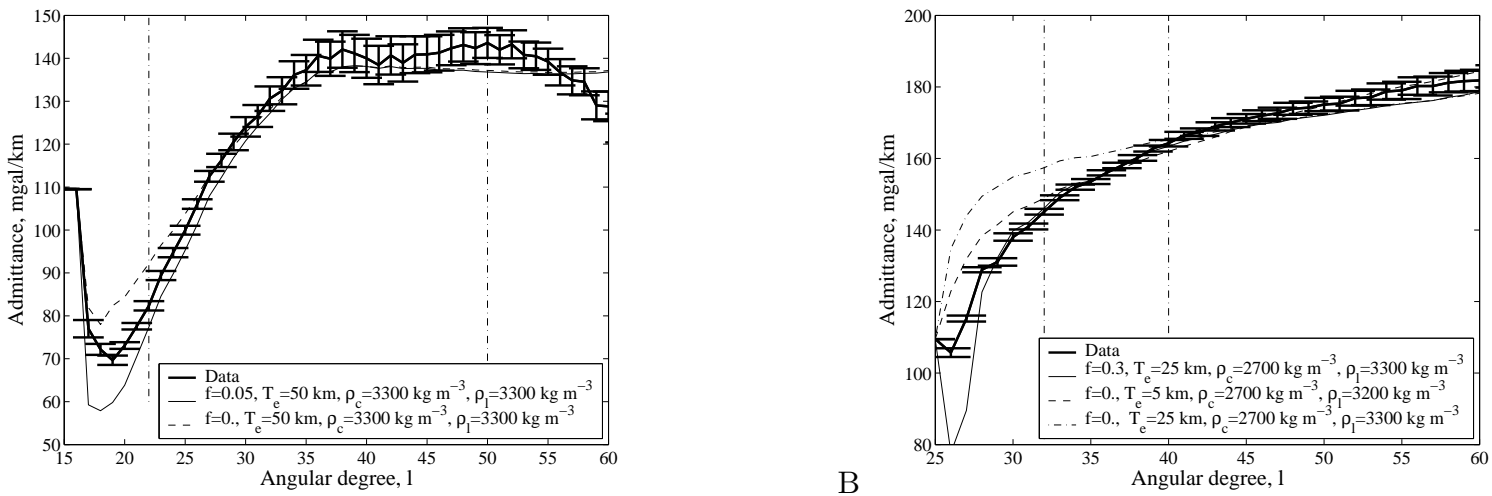

Figure 10. (A) Observed and best theoretical admittances for Elysium when $f=0$ and $f=0.05$. (B)

The same for Arsia with $f=0$ and $f=0.3$.

Table 3. Summary of the results and comparison with McGovern et al. [2004]

\begin{tabular}{lcccccccc}
\hline \multirow{2}{*}{ Volcano } & \multicolumn{4}{c}{ This study } & \multicolumn{4}{c}{ McGovern et al. $[2004]$} \\
\cline { 2 - 8 } & $\theta_{0}$ & $\rho_{l}\left(\mathrm{~kg} \mathrm{~m}^{-3}\right)$ & \multicolumn{2}{c}{$T_{e}(\mathrm{~km})$} & $\theta_{0}$ & $\rho_{l}\left(\mathrm{~kg} \mathrm{~m}^{-3}\right)$ & $T_{e}(\mathrm{~km})$ \\
& & $\mathrm{f}=0$ & $\mathrm{f} \neq 0$ & $\mathrm{f}=0$ & $\mathrm{f} \neq 0$ & & & \\
\hline Elysium & $15^{\circ}$ & $3210 \pm 180$ & $>2900$ & $56 \pm 20$ & $<175$ & $21^{\circ}$ & 3250 & $15-45$ \\
Olympus & $15^{\circ}$ & $3194 \pm 110$ & $3252 \pm 150$ & $93 \pm 40$ & $>70$ & $21^{\circ}$ & 3150 & $>70$ \\
Alba Patera & $15^{\circ}$ & $<3100$ & $<3300$ & $66 \pm 20$ & $73 \pm 30$ & $21^{\circ}$ & 2950 & $38-65$ \\
Pavonis & $10^{\circ}$ & $3266 \pm 120$ & - & $>50$ & $>50$ & $15^{\circ}$ & 3250 & $<100$ \\
Arsia & $10^{\circ}$ & $3240 \pm 130$ & $3300 \pm 100$ & $<30$ & $<35$ & $15^{\circ}$ & 3250 & $2-80$ \\
Ascraeus & $10^{\circ}$ & $3200 \pm 100$ & $>2900$ & $105 \pm 40$ & $>50$ & $15^{\circ}$ & 3300 & $>20$ \\
\hline
\end{tabular}

order to model the low-degree admittance spectra for this volcano.

\subsubsection{Constraints on the crustal density and the crustal thickness}

Figure 8 shows that the crustal density is only constrained for the Elysium rise with a value of $\rho_{c}=3270 \pm 150 \mathrm{~kg} \mathrm{~m}^{-3}$. However, even if less constrained, similar high values of crustal density for Olympus Mons and Alba Patera seems to be implied. There is a $98 \%$ probability that $\rho_{c}>3000$ $\mathrm{kg} \mathrm{m}{ }^{-3}$ for Olympus Mons, whereas for Alba Patera the minimum $\chi^{2} / \nu$ corresponds to $\rho_{c}=3300 \mathrm{~kg} \mathrm{~m}^{-3}$. Figure 9 shows that the crustal thickness is not constrained for any of the volcanoes. These results are summarized and compared with those of McGovern et al. [2004] in Table 3.

\subsection{Top and bottom loads results}
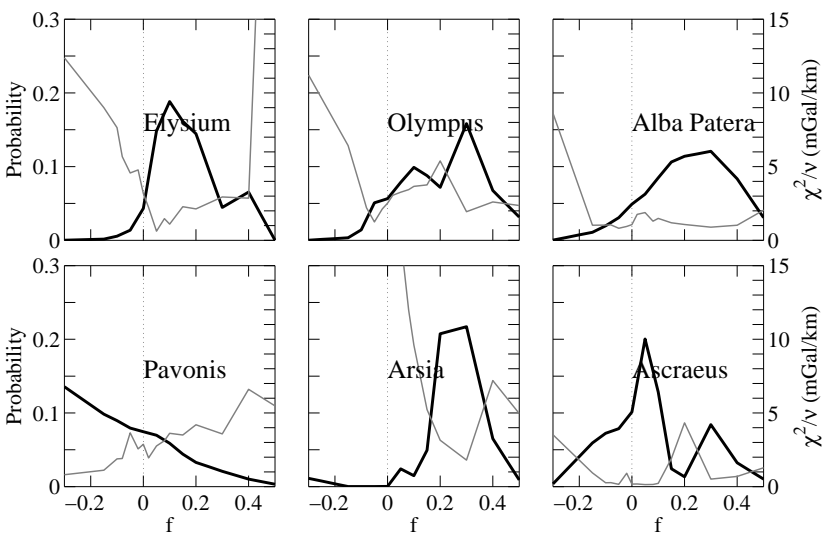

Figure 11. Left axis: Marginal probability as a function of $f$. Right axis: Minimum $\chi^{2} / \nu$. The vertical dotted line highlights the case $f=0$.
In this section, we explore the consequence of subsurface density anomalies within either the crust or mantle (see Figure 1). As the above results show that the crustal thickness is not constrained, and since a full exploration of the five dimensional model space is computationally infeasible, we chose here to keep this parameter fixed with a value of $T_{c}=$ $50 \mathrm{~km}$ [e.g., Wieczorek and Zuber, 2004]. All other parameters were varied according to the values listed in Table 2. Figure 11 displays the minimum $\chi^{2} / \nu$ and marginal probability as a function of $f$. In general, the inclusion of subsurface loads tends to decrease the value of the minimum $\chi^{2} / \nu$ as a result of a better fit to the lowest angular degrees of the admittance function. For Elysium and Arsia, which were poorly modeled in the case of surface-only loads, the addition of subsurface loads in the mantle considerably improved the models, with values of $\frac{\chi_{\min }^{2}}{\nu}$ now being close to unity. Figure 10 shows the improvement of the theoretical admittance when subsurface loads are added. The most probable value of $f$ for Elysium is $f=0.1 \pm 0.06$ and for Arsia, $f=0.25 \pm 0.1$. By definition, a positive value of $f$ corresponds to the presence of less dense material in the mantle which could be due to a higher temperature and/or a depleted mantle composition.

For the other volcanoes, Figure 11 shows that the value of $f$ is less well constrained. Nevertheless, some trends exist and better results are generally obtained for $f>0$. For Olympus Mons and Alba Patera, the marginal probability gives respectively $f=0.22 \pm 0.16$ and $f=0.2 \pm 0.16$. For Pavonis, the marginal probability increases for $f<0$, suggesting the presence of more dense material located in the crust. For Ascraeus, the distribution is irregular, but shows preferred values near $f=0$.

The inclusion of subsurface loads tends, in general, to enlarge the uncertainties on the other parameters. With the exception of Alba Patera, the load densities are still constrained to be about $3200 \mathrm{~kg} \mathrm{~m}^{-3}$, although the uncertainty is now much larger $\left(\sim 200 \mathrm{~kg} \mathrm{~m}^{-3}\right.$ compared to $\sim 100 \mathrm{~kg} \mathrm{~m}^{-3}$ found in the previous section, see Table 3 ). The most important change is for the elastic thickness, where only an upper 
or lower bound is obtained as result of the addition of $f$ as a model parameter. The sole exception is Alba Patera where it is found to be $73 \pm 30 \mathrm{~km}$ (both the mean value and error are slightly larger than the values obtained assuming $f=0$ ). For Elysium and Arsia Montes, our results give only an upper limit, with $T_{e}<150$ and $T_{e}<50 \mathrm{~km}$ respectively. For Olympus, Pavonis and Ascraeus Montes, only values lower than $50 \mathrm{~km}$ are excluded. For the crustal density, results are similar to those obtained with only surface loads, with only the density for Elysium Mons being clearly contrained with a value $\sim 3300 \mathrm{~kg} \mathrm{~m}^{-3}$.

\section{Discussion}

For all volcanoes studied here, with the exception of Alba Patera, we find that the best constrained parameter is the load density with a value of $\rho_{l}=3200 \pm 100 \mathrm{~kg} \mathrm{~m}^{-3}$. This is considerably larger than previously published values of about $2900 \mathrm{~kg} \mathrm{~m}^{-3}$ [McGovern et al., 2002; McKenzie et al., 2002], but is consistent with the corrected values of $\mathrm{Mc}$ Govern et al. [2004] (see Table 3 for a comparison). These high densities are in agreement with those of the Martian basaltic meteorites, which are believed to come either from the Tharsis or Elysium regions [e.g. McSween, 1985; Head et al., 2002]. As shown in Neumann et al. [2004], bulk porefree densities of these meteorites which were calculated from modal mineral abundances lie between 3220 and $3390 \mathrm{~kg}$ $\mathrm{m}^{-3}$. While few in situ density measurements of the Martian meteorites are available [Britt and Consolmagno, 2003], they are consistent with the values of Neumann et al. [2004]. A typical value for the porosity of the Martian meteorites is $\sim 5 \%$ [Britt and Consolmagno, 2003] and this would lead to a reduction of their calculated densities by $\sim 150 \mathrm{~kg} \mathrm{~m}^{-3}$ if the pore space was filled with air. If instead the pore space was filled with liquid water or ice, then the bulk density would only be reduced by $\sim 100 \mathrm{~kg} \mathrm{~m}^{-3}$. For comparison, we note that the porosity of Hawaiian lavas are typically less than $5 \%$ for depths greater than $1 \mathrm{~km}$ [Moore, 2001]. After accounting for a reasonable density reduction of 100 $\mathrm{kg} \mathrm{m}^{-3}$, the density range for the Martian meteorites (3120$3290 \mathrm{~kg} \mathrm{~m}^{-3}$ ) is found to be nearly identical to the values obtained from this geophysical study $\left(3200 \pm 100 \mathrm{~kg} \mathrm{~m}^{-3}\right)$. As the load density is relatively constant for the volcanoes studied here, this suggests that similar magmatic process have operated at each of these regions. The lower density obtained for Alba Patera (less than $3100 \mathrm{~kg} \mathrm{~m}^{-3}$ ) might imply that its composition is less iron-rich than the known Martian meteorites. Alternatively, given the low coherences for this volcano at high degrees, it is possible that uncorrelated subsurface loads might be important for this volcano, and that neglecting such a process could have biased our results there [Forsyth, 1985, e.g.,].

Our elastic thickness estimates are found to be consistent with the revised values of McGovern et al. [2004] within their respective uncertainties (see Table 3). Nevertheless, we note that our best fit values are generally larger than those of McGovern et al. [2004] and our uncertainties are considerably different. For instance, only a lower bound on the elastic thickness for Olympus Mons was obtained in their study $\left(T_{e}>70 \mathrm{~km}\right)$, whereas our estimate is somewhat better constrained $(93 \pm 40 \mathrm{~km})$. Furthermore, while they obtained only an upper bound for Pavonis Mons $\left(T_{e}<100\right.$ $\mathrm{km}$ ), we find that the elastic thickness is not constrained for this volcano.

Several possibilities could explain the differences between our study and that of McGovern et al. [2004]. First, this could be in part a result of our more accurate technique for calculating the load acting on the lithosphere. Figure 5 demonstrates that the various approximations employed in previous studies could lead to significant errors in the modeled gravity anomaly. In particular, we have shown that the largest differences occur for low values of $T_{e}$, and when $\rho_{l}$ differs from $\rho_{c}$. Second, we have computed functional misfits for a four-dimensional parameter space, whereas in $\mathrm{Mc}$ Govern et al. [2002], misfits were calculated only as a function of two parameters while keeping the other two fixed. Many parameters in elastic thickness models are correlated, and by adding more degrees of freedom to the inversion it should come as no surprise that fewer parameters will be constrained and that their respective uncertainties will be larger [see also Lawrence and Phillips, 2003]. Third, we have used a better localization window when calculating the localized admittance and coherence functions. Our windows concentrate $\sim 99 \%$ of their energy within the region of interest in comparison to that of Simons et al. [1997] that is only concentrated at $\sim 93 \%$. Finally, it is worth mentioning that our window size is systematically smaller than that used in McGovern et al. [2002] by about $5^{\circ}\left(\theta_{0}=15^{\circ}\right.$ vs. $21^{\circ}$, or $\theta_{0}=10^{\circ}$ vs. $\left.15^{\circ}\right)$. The choice of the window diameter is a rather subjective choice and window sizes were chosen here to minimize the contribution from the Utopia mascon to the Elysium rise, the Tharsis contribution to Olympus Mons or Alba Patera, and to better isolate the individual Tharsis Montes. We have checked that a larger window size yields similar results for Elysium, Olympus Mons and Alba Patera.

It should be emphasized, that the large uncertainties associated with the elastic thickness of the Martian volcanoes will hinder any attempt to constrain how this parameter varies in both space and time, and will likely reduce the utility of heat flow estimates which can be derived from these values [e.g. McNutt, 1984; Solomon and Head, 1990; McGovern et al., 2002]. This result is considerably amplified when subsurface loads are taken into account.

Based on the lack of circumferential grabens around Olympus Mons, Thurber and Toksoz [1978] have argued that little lithospheric deflection has occurred beneath this volcano, and that the regional elastic thickness must hence be greater than $150 \mathrm{~km}$. In contrast, our value is somewhat smaller $(93 \pm 40 \mathrm{~km})$, seemingly at odds with the tectonic constraints. Comer et al. [1985] have investigated whether viscous relaxation might modify this constraint, but concluded that an elastic support model with an elastic thickness of $\sim 200 \mathrm{~km}$ was most likely. Nevertheless, as discussed in Comer et al. [1985], alternative mechanism might act to reduce or obscure circumferential faulting around this volcano. For instance, a global thermal compressive state of the planet could have suppressed the formation of extensional fractures, or fault burial could have occurred given the large number of relatively young volcanic flows that have resurfaced large portions of the surrounding region. Dynamic support might also help to minimize the extensional stresses in the region. In support of this latter suggestion, we have highlighted the likely presence of less dense material in the mantle underlying Olympus Mons, possibly related to an active plume. It is also possible that a more sophisticated elastoviscoplastic model developed in spherical coordinates might yield slightly different results [see Freed et al., 2001; Albert et al., 2000].

We have found that all parameters except $T_{c}$ are well constrained for the Elysium rise. We note that this result might be related to the fact that this feature is relatively isolated and far from the Tharsis plateau, whereas the other volcanoes are very close to each other and are difficult to isolate in the space domain. For the Elysium region of Mars, the crustal density is predicted to be similar to the load density with a value of $3270 \pm 150 \mathrm{~kg} \mathrm{~m}^{-3}$. Given the similarity between the crustal density, the load density and the Martian meteorites, it is possible that the crustal composition of the Northern lowlands, like the volcanic edifices, is similar 
to these meteorites. While no strong constraints exist regarding the average crustal density of either the northern os southern hemispheres of Mars, one possible constraint comes from the composition of rocks measured in situ at the Mars Pathfinder site. This landing site is located in an outflow channel that originates within the highlands and therefore rocks found there could have an origin from the ancient highlands of Mars. Neumann et al. [2004] have calculated the modal mineralogy of the Mars Pathfinder "soil free rock" [Brückner et al., 2003] and have determined that its porefree density is about $3060 \mathrm{~kg} \mathrm{~m}^{-3}$. If this value is representative of the southern highlands crust, and if our results for the Elysium rise are typical of the northern hemisphere, then this implies that the composition of the northern hemisphere crust is considerably different than that of the southern highlands. In particular, the Northern lowlands would be more dense by $\sim 200 \mathrm{~kg} \mathrm{~m}^{-3}$. This suggests the possibility that Pratt compensation (i.e, lateral density variations as opposed to crustal thickness variations) may be largely responsible for the 3.1-km center-of-mass/center-of-figure offset of the planet. Assuming an average crustal thickness between 38 and $62 \mathrm{~km}$ [Wieczorek and Zuber, 2004], the $\sim 6$ $\mathrm{km}$ difference in elevation between the Northern lowlands and Southern highlands would correspond to a difference in density between $\sim 310$ and $520 \mathrm{~kg} \mathrm{~m}^{-3}$. Though slightly on the high side, this number is roughly consistent with the difference in density between the Mars Pathfinder soil free rock (assumed to be representative of the Southern highlands) and the crust beneath the Elysium rise.

Given the early Noachian age of the Martian lowlands [Frey et al., 2002], this density and compositional dichotomy between the two hemispheres must have formed during the earliest geologic evolution of the planet. One possible origin is an early episode of plate tectonics operating in the Northern lowlands. In this scenario, the Southern highlands of Mars could either represent an ancient primordial crust or the resulting products of subduction related volcanism. Alternatively, it is possible that this feature could be related to a fundamental asymmetry in the primary differentiation of this planet. For example, the nearside-farside dichotomy of the Moon appears to be related to the asymmetric crystallization of a near global magma ocean [e.g. Jolliff et al., 2000].

Lastly, we emphasize that the inclusion of subsurface loads dramatically improves the model fits for the Elysium rise and Arsia Mons, where less dense materials in the mantle are required to explain the gravity and topography data. Whereas model results for Olympus Mons and Alba Patera are not noticeably improved by the addition of subsurface loads, a less dense mantle is nevertheless preferred to dense crustal intrusions. A less dense mantle beneath these volcanoes can be interpreted as being a result of thermal and/or compositional effects. In the latter case, the extraction of dense iron-rich basaltic magmas from the mantle would act to decrease its density [Oxburgh and Parmentier, 1977] and the contribution of these two effects can be parameterized by the relation

$$
\Delta \rho=-\rho_{m}[\beta F+\alpha \Delta T],
$$

where $\Delta \rho$ and $\Delta T$ are respectively the density and the temperature anomalies within the mantle, $\rho_{m}$ the mean mantle density (see Table 2), $\alpha$ is the thermal expansion coefficient (here assumed to be $3 \times 10^{-5}{ }^{\circ} \mathrm{C}^{-1}$ ), $F$ is the degree of depletion and $\beta$ is a coefficient of density reduction due to the extraction of partial melts. In order to quantify roughly the expected density variation due to compositional effects, we set $\beta=0.07$ [e.g. Jordan, 1979] and use the interval $0.02 \leq F \leq 0.12$ obtained by Kiefer [2003], yielding an expected density variation of $\sim 5-30 \mathrm{~kg} \mathrm{~m}^{-3}$. On the other hand, convective modeling of Kiefer [2003] suggest that the plume conduit beneath the Tharsis rise could be as much as $\sim 400^{\circ} \mathrm{C}$ greater than the ambient mantle. This temperature variation would correspond to a density variation of $\sim 40 \mathrm{~kg} \mathrm{~m}^{-3}$. Combining these two effects, we would expect a density variation less than $70 \mathrm{~kg} \mathrm{~m}^{-3}$.

We next compare the above expected density variations with the magnitude of the subsurface load obtained from our admittance analysis. The density variation from our models is derived from equation 34 for a given a value of $f$, the thickness of the "plume" $M$, and the thickness of the surface load $h_{l}$. Given the computational constraints, only one value of $M$ was used in our subsurface loading model $(M=250$ $\mathrm{km}$ ), and it should be clear that an increase (decrease) of this parameter would decrease (increase) the obtained density anomaly by nearly the same factor. For the Elysium rise, $\mathrm{f}=0.1 \pm 0.06$, and the associated density variations are predicted to lie between 10 and $45 \mathrm{~kg} \mathrm{~m}^{-3}$. This can be explained by either a thermal or compositional effect. For Arsia, $\mathrm{f}=0.25 \pm 0.1$, and this yields rather large density variations between 170 and $390 \mathrm{~kg} \mathrm{~m}^{-3}$. Even the lowest of these values is much larger than the expected variations cited above, possibly implying higher temperatures and/or a higher degree of mantle depletion beneath this volcano. This might not be an unreasonable expectation as Arsia Mons is the most prominent of the Tharsis Montes, and could be located directly above an active plume (the other Tharsis Montes would be off the plume axis in this scenario). Even if the value of $f$ is not as well defined for the other volcanoes, positive values are preferred for Olympus Mons, and the variation in density is found to lie between 0 and $230 \mathrm{~kg}$ $\mathrm{m}^{-3}$, consistent with thermal and/or composition effects.

The negative density anomalies beneath the major volcanoes is most reasonably interpreted as being the result of a mantle plume. The results of this study thus imply that the mantle is not only dynamically active in the region of Tharsis and Olympus Mons, but also beneath the Elysium rise. A currently active plume is consistent with recent analyses of cratering statistics on Olympus Mons and the Elysium rise which suggest the presence of young lava flows $[\sim 10-30$ Myr, Hartmann and Neukum, 2001] and with the radiometric ages of the Shergottites which posses crystalization ages between 175 and 475 Myr [Nyquist et al., 2001].

For Pavonis, we found that preferred models are obtained for $f<0$. If our admittance model is correct for this volcano, then this implies the presence of dense intrusive materials in the crust, such as the differentiation products associated with a central magma chamber.

\section{Conclusions}

We have presented here an accurate method to analyze localized gravity/topography admittances of the major Martian volcanoes by assuming that surface and subsurface loads are elastically supported by the lithosphere. Our analysis represents an improvement over previous studies in several ways. First, our methodology computes the gravity anomaly, surface deflection, and load acting on the lithosphere in a self-consistent manner. Previous studies have not been able to correctly model the case where the load density differs from that of the crust. Secondly, we calculate localized admittance and coherence spectra using localizing windows that concentrate almost all of their energy $(\sim 99 \%)$ within the region of interest, whereas previous studies have employed sub-optimal windows that are only concentrated at about $93 \%$. Finally we have systematically investigated the misfit function for the high dimensional space which includes the elastic thickness, crustal thickness, load density, crustal density, and ratio of subsurface to surface loading. The results we have here obtained, although consistent with 
those of McGovern et al. [2004], offers more precise and reliable bounds on the elastic thickness and load density.

The main result we have obtained is the density of the volcanoes. With the exception of Alba Patera, we have obtained a value of $3200 \pm 100 \mathrm{~kg} \mathrm{~m}^{-3}$ that is higher than what was previously published (i.e. $2900 \pm 100 \mathrm{~kg} \mathrm{~m}^{-3}[$ McKenzie et al., 2002; McGovern et al., 2002]), but consistent with the corrected value of McGovern et al. [2004]. This high densities are in agreement with those of the Martian basaltic meteorites, which are believed to come from the Tharsis or Elysium volcanic province. When the subsurface load are neglected, the elastic thickness is found to be moderately constrained for Elysium $(56 \pm 20 \mathrm{~km})$, Alba Patera $(66 \pm 20 \mathrm{~km})$, Olympus Mons $(93 \pm 40 \mathrm{~km})$ and Ascraeus $(105 \pm 105 \mathrm{~km})$. However, when subsurface loads are taken into account, the uncertainties obtained are then extremely large, with the exception of Alba Patera where we obtain $T_{e}=73 \pm 30 \mathrm{~km}$. The crustal density is only constrained for the Elysium region $\left(\rho_{c}=3270 \pm 150 \mathrm{~kg} \mathrm{~m}^{-3}\right)$. Given the similarity among the crustal density, load density and Martian meteorites, it is possible that the crustal composition of the Northern lowlands is similar to these meteorites. Estimates for the density of the Southern highlands crust are generally lower [Neumann et al., 2004], and this seems to indicate that the northern hemisphere crust is more mafic in composition. Such a difference suggests the possibility that Pratt compensation may be partially responsible for the $3.1 \mathrm{~km}$ center of mass/center of figure offset of the planet.

Finally, we have shown that subsurface loads play an important role in the gravity and topography signal for the Elysium rise and Arsia Mons, and to a lesser extent Olympus Mons and Alba Patera. The less dense mantle beneath these volcanoes is most reasonably interpreted as an active plume which contains contribution from both thermal and compositional effects. Such an assessment is consistent with recent volcanism in this region as implied by crater counting studies, as well as the ages of the basaltic Martian meteorites.

\section{Appendix A: mapped coordinates}

In this appendix, we show how equations 1 and 2 can be expressed in terms of the mapped coordinates $s, \theta, \phi$. First, by differentiating equation 3 we obtain:

$$
d r=a_{1} d s+a_{2} d \theta+a_{3} d \phi
$$

where

$a_{1}=\frac{\partial r}{\partial s}=\frac{r_{i+1}(\theta, \phi)-r_{i}(\theta, \phi)}{s_{i+1}-s_{i}}$

$a_{2}=\frac{\partial r}{\partial \theta}=\left(\frac{s-s_{i}}{s_{i+1}-s_{i}}\right) \frac{\partial r_{i+1}(\theta, \phi)}{\partial \theta}+\left(\frac{s_{i+1}-s}{s_{i+1}-s_{i}}\right) \frac{\partial r_{i}\left(\theta, \phi_{0}\right.}{\partial \theta}$ $a_{3}=\frac{\partial r}{\partial \phi}=\left(\frac{s-s_{i}}{s_{i+1}-s_{i}}\right) \frac{\partial r_{i+1}(\theta, \phi)}{\partial \phi}+\left(\frac{s_{i+1}-s}{s_{i+1}-s_{i}}\right) \frac{\partial r_{i}(\theta \phi \phi)}{\partial \phi} \frac{\partial U}{\partial s}=\frac{r_{i+1}-r_{i}}{s_{i+1}-s_{i}} G_{r}$

The transformation matrix between $(d r, d \theta, d \phi)$ and $(d s$, $d \theta, d \phi)$ can be written as follows in matrix notation

$$
\left[\begin{array}{l}
d s \\
d \theta \\
d \phi
\end{array}\right]=\left[\begin{array}{ccc}
1+b_{1} & b_{2} & b_{3} \\
0 & 1 & 0 \\
0 & 0 & 1
\end{array}\right]\left[\begin{array}{l}
d r \\
d \theta \\
d \phi
\end{array}\right]
$$

where

$$
\begin{aligned}
& b_{1}=\frac{1}{a_{1}}-1 \\
& b_{2}=-\frac{a_{2}}{a_{1}} \\
& b_{3}=-\frac{a_{3}}{a_{1}} .
\end{aligned}
$$

Applying the chain rule for partial derivatives of a function $f=f(r, \theta, \phi)$ where $r$ is a function of $s, \theta$ and $\phi$ yields

$$
\begin{aligned}
& \frac{\partial f}{\partial r}=\frac{\partial f}{\partial s} \frac{\partial s}{\partial r}+\frac{\partial f}{\partial \theta} \frac{\partial \theta}{\partial r}+\frac{\partial f}{\partial \phi} \frac{\partial \phi}{\partial r} \\
& \frac{\partial f}{\partial \theta}=\frac{\partial f}{\partial s} \frac{\partial s}{\partial \theta}+\frac{\partial f}{\partial \theta} \\
& \frac{\partial f}{\partial \phi}=\frac{\partial f}{\partial s} \frac{\partial s}{\partial \phi}+\frac{\partial f}{\partial \phi}
\end{aligned}
$$

Using equation $\mathrm{A} 5$, we then obtain then

$$
\begin{aligned}
& \frac{\partial f}{\partial r}=\left(1+b_{1}\right) \frac{\partial f}{\partial s} \\
& \frac{\partial f}{\partial \theta}=b_{2} \frac{\partial f}{\partial s}+\frac{\partial f}{\partial \theta} \\
& \frac{\partial f}{\partial \phi}=b_{3} \frac{\partial f}{\partial s}+\frac{\partial f}{\partial \phi}
\end{aligned}
$$

The relationship between the derivative operators in the two sets of coordinates can be shown to be:

$$
\left[\begin{array}{c}
\frac{\partial}{\partial r} \\
\frac{\partial}{\partial \theta} \\
\frac{\partial}{\partial \phi}
\end{array}\right]=\mathbf{A}\left[\begin{array}{c}
\frac{\partial}{\partial s} \\
\frac{\partial}{\partial \theta} \\
\frac{\partial}{\partial \phi}
\end{array}\right]
$$

where $\mathbf{A}$ is defined by

$$
\mathbf{A}=\left[\begin{array}{ccc}
1+b_{1} & 0 & 0 \\
b_{2} & 1 & 0 \\
b_{3} & 0 & 1
\end{array}\right]
$$

Equation 1 can be rewritten in a matrix form as

$$
\left[\begin{array}{l}
G_{r} \\
G_{\theta} \\
G_{\phi}
\end{array}\right]=\left[\begin{array}{r}
\frac{\partial}{\partial r} \\
\frac{1}{r} \frac{\partial}{\partial \theta} \\
\frac{1}{r \sin \theta} \frac{\partial}{\partial \phi}
\end{array}\right] U
$$

which can be rewritten in the new set of coordinates $(s, \theta, \phi)$ using the transformation matrix $\mathbf{A}$ as

$$
\left[\begin{array}{c}
G_{r} \\
G_{\theta} \\
G_{\phi}
\end{array}\right]=\left[\begin{array}{ccc}
1+b_{1} & 0 & 0 \\
\frac{b_{2}}{r} & \frac{1}{r} & 0 \\
\frac{b_{3}}{r \sin \theta} & 0 & \frac{1}{r \sin \theta}
\end{array}\right]\left[\begin{array}{c}
\frac{\partial U}{\partial s} \\
\frac{\partial U}{\partial \theta} \\
\frac{\partial U}{\partial \phi}
\end{array}\right] .
$$

$G_{\alpha}=D_{\alpha} U$

$$
-\left[\frac{s-s_{i}}{r_{i+1}-r_{i}} D_{\alpha} r_{i+1}+\frac{s_{i+1}-s}{r_{i+1}-r_{i}} D_{\alpha} r_{i}\right] \frac{\partial U}{\partial s}
$$

where $\alpha$ corresponds to the horizontal coordinates $\theta$ or $\phi$ and where $D_{\alpha}$ is defined by

$$
D_{\alpha}= \begin{cases}\frac{1}{r} \frac{\partial}{\partial \theta} & \text { if } \alpha=\theta \\ \frac{1}{r \sin \theta} \frac{\partial}{\partial \phi} & \text { if } \alpha=\phi\end{cases}
$$

In order to rewrite equation 2 in a matrix form, it will be convenient to start in Cartesian coordinates. The relationship between the Cartesian and spherical components of the 
gravity field is given by

$$
\left[\begin{array}{l}
G_{x} \\
G_{y} \\
G_{z}
\end{array}\right]=M\left[\begin{array}{l}
G_{r} \\
G_{\theta} \\
G_{\phi}
\end{array}\right]
$$

where

$$
M=\left[\begin{array}{lll}
\sin \theta \cos \phi & \cos \theta \cos \phi & -\sin \phi \\
\sin \theta \sin \phi & \cos \theta \sin \phi & \cos \phi \\
\cos \theta & -\sin \theta & 0
\end{array}\right]
$$

Equation 2 can then be written as

$$
\begin{aligned}
& \nabla \cdot \mathbf{G}=-4 \pi \mathcal{G} \rho \\
& =\left[\begin{array}{lll}
\frac{\partial}{\partial x} & \frac{\partial}{\partial y} & \frac{\partial}{\partial z}
\end{array}\right]\left[\begin{array}{l}
G_{x} \\
G_{y} \\
G_{z}
\end{array}\right] \\
& =\left(\mathbf{M}\left[\begin{array}{ccc}
1+b_{1} & 0 & 0 \\
\frac{b_{2}}{r} & \frac{1}{r} & 0 \\
\frac{b_{3}}{r \sin \theta} & 0 & \frac{1}{r \sin \theta}
\end{array}\right]\left[\begin{array}{c}
\frac{\partial}{\partial s} \\
\frac{\partial}{\partial \theta} \\
\frac{\partial}{\partial \phi}
\end{array}\right]\right)^{t} \mathbf{M}\left[\begin{array}{c}
G_{r} \\
\left(\mathrm{~A}_{2} 26\right. \\
G_{\phi}
\end{array}\right] \\
& =\frac{\partial G_{r}}{\partial s}+\frac{2 G_{r}}{r}+\frac{1}{r \sin \theta}\left[\frac{\partial}{\partial \theta}\left(\sin \theta G_{\theta}\right)+\frac{\partial G_{\phi}}{\partial \phi}(27)\right. \\
& +\left(\mathbf{M}\left[\begin{array}{c}
b_{1} \\
\frac{b_{2}}{r} \\
\frac{b_{3}}{r \sin \theta}
\end{array}\right]\right)^{t} \mathbf{M}\left[\begin{array}{c}
\frac{\partial G_{r}}{\partial s} \\
\frac{\partial G_{\theta}}{\partial s} \\
\frac{\partial G_{\phi}}{\partial s}
\end{array}\right] \\
& =\frac{\partial G_{r}}{\partial s}+\frac{2 G_{r}}{r}+\frac{1}{r \sin \theta}\left[\frac{\partial}{\partial \theta}\left(\sin \theta G_{\theta}\right)+\frac{\partial G_{\phi}}{\partial \phi}(28)\right. \\
& +\left[\begin{array}{lll}
b_{1} & \frac{b_{2}}{r} & \frac{b_{3}}{r \sin \theta}
\end{array}\right]\left[\begin{array}{c}
\frac{\partial G_{r}}{\partial s} \\
\frac{\partial G_{\theta}}{\partial s} \\
\frac{\partial G_{\phi}}{\partial s}
\end{array}\right] .
\end{aligned}
$$

(1)

The spherical harmonic transform and reconstruction of an

$$
\begin{aligned}
u_{\ell m} & =\frac{1}{4 \pi} \int_{\Omega} u(\theta, \phi) Y_{\ell}^{m^{*}}(\theta, \phi) d \Omega \\
u(\theta, \phi) & =\sum_{\ell=0}^{\infty} \sum_{m=-\ell}^{\ell} u_{\ell m} Y_{\ell}^{m}(\theta, \phi) .
\end{aligned}
$$

Substituting B2 into B1 we obtain the expression

$$
U(r, \theta, \phi)=\sum_{\ell=0}^{\infty} \sum_{m=-\ell}^{\ell}\left(\frac{1}{r}\right)^{\ell+1} \frac{1}{2 \ell+1} \xi_{\ell m} Y_{\ell}^{m}(\theta, \phi),
$$

where

$$
\xi_{\ell m}=\int_{\Omega^{\prime}} \int_{r_{i}\left(\theta^{\prime}, \phi^{\prime}\right)}^{r_{i+1}\left(\theta^{\prime}, \phi^{\prime}\right)} \mathcal{G} \rho\left(r^{\prime}, \theta^{\prime}, \phi^{\prime}\right)\left(r^{\prime}\right)^{\ell+2} d r^{\prime} Y_{\ell}^{m^{*}}\left(\theta^{\prime}, \phi^{\prime}\right) d \Omega^{\prime}
$$

which is valid for all points exterior to the planet. In order to work on spherical integration boundaries, we use the set of coordinates $s, \theta, \phi$ as defined by equation 3 . After a change of variables, $\xi_{\ell m}$ can be expressed as

$$
\xi_{\ell m}=\int_{\Omega^{\prime}} \int_{s_{i}}^{s_{i+1}} \mathcal{G} \rho\left(s, \theta^{\prime}, \phi^{\prime}\right)\left(r^{\prime}\right)^{\ell}\left|J^{\prime}\right| s^{2} d s Y_{\ell}^{m^{*}}\left(\theta^{\prime}, \phi^{\prime}\right) d \Omega^{\prime},
$$

where $\left|J^{\prime}\right|$ is defined as $\frac{r^{\prime 2}}{s^{2}}|J|$, and $|J|$ is the Jacobian determinant, $|J|=\left|\frac{\partial(r, \theta, \phi)}{\partial(s, \theta, \phi)}\right|^{s^{2}}=\left|\frac{r_{i+1}(\theta, \phi)-r_{i}(\theta, \phi)}{s_{i+1}-s_{i}}\right|$.

The integral over $s$ can be computed by Gauss-Legendre quadrature on a set of grid points $\left(\theta_{i}, \phi_{i}\right)$ and then the integral over $\theta$ and $\phi$ can be calculated using standard spherical harmonic transform routines [e.g., Lognonné and Romanowicz [1990], Appendix 5; Driscoll and Healy [1994]; Sneeuw [1994]]. To facilitate this latter operation, the grid coordinates $\left(\theta_{i}, \phi_{i}\right)$ are chosen to be equally spaced in longitude and with latitude points corresponding to the zeros of the Legendre polynomials $P_{2 \mathrm{~L}_{\max }-1}$, where $L$ is the maximum degree of the spherical harmonic transform.

\section{Appendix C: Generalized spherical harmonics}

In order to use properties similar to those of the spherical harmonics when investigating vector (or tensor) fields, we introduce here the generalized spherical harmonics, as

$$
U(r, \theta, \phi)=\int_{V^{\prime}} \frac{\mathcal{G} \rho\left(\overrightarrow{\mathbf{r}^{\prime}}\right) d V^{\prime}}{\left|\overrightarrow{\mathbf{r}}-\overrightarrow{\mathbf{r}^{\prime}}\right|}
$$


described in Phinney and Burridge [1973]. If the vector $\mathbf{u}(r, \theta, \phi)$ has components $u_{r}, u_{\theta}$ and $u_{\phi}$, it is convenient to define the new variables

$$
\begin{aligned}
u^{-} & =\frac{1}{\sqrt{2}}\left(u_{\theta}+i u_{\phi}\right) \\
u^{0} & =u_{r} \\
u^{+} & =\frac{1}{\sqrt{2}}\left(-u_{\theta}+i u_{\phi}\right) .
\end{aligned}
$$

These components can then be expanded in terms of generalized spherical harmonics as

$$
u^{\alpha}(r, \theta, \phi)=\sum_{\ell=0}^{\infty} \sum_{m=-\ell}^{\ell} u_{\ell}^{\alpha, m} Y_{\ell}^{N, m}(\theta, \phi),
$$

where $Y_{\ell}^{N, m}(\theta, \phi)$ are the generalized spherical harmonics, $u_{\ell}^{\alpha, m}$ are the generalized spherical harmonics coefficients and $N$ is equal to $-1,0$, and 1 when $\alpha$ is -, 0 , and + , respectively. The generalized spherical harmonics are defined as

$$
Y_{\ell}^{N, m}(\theta, \phi)=P_{\ell}^{N, m}(\cos \theta) e^{i m \phi},
$$

where $P_{\ell}^{N, m}$ is the generalized Legendre functions. We note that when $N=0, P_{\ell}^{0, m}$ is equal to the standard associated Legendre functions $P_{\ell}^{m}$. Recurrence algorithms for calculating these can be found in Phinney and Burridge [1973]. Expressions for the gradient and divergence are given in appendix C of Phinney and Burridge [1973], and we here quote the relevant equations. The gradient of a scalar $U(r, \theta, \phi)$ is given by:

$$
\begin{aligned}
D_{\alpha} U(r, \theta, \phi) & =\frac{1}{r} \sqrt{\frac{\ell(\ell+1)}{2}} U_{\ell m}(r) Y_{\ell}^{\alpha, m}(\theta, \phi), \\
D_{0} U(r, \theta, \phi) & =\frac{d}{d r} U_{\ell m}(r) Y_{\ell}^{0, m}(\theta, \phi),
\end{aligned}
$$

where $\alpha=-1$ or 1 . The divergence of a vector $\mathbf{G}(r, \theta, \phi)$ can be expressed in this basis by:

$$
\begin{aligned}
\nabla \cdot \mathbf{G}(r, \theta, \phi) & =\left\{\left(\frac{d}{d r}+\frac{2}{r}\right) G_{\ell m}^{0}(r)\right. \\
& \left.-\frac{1}{r} \sqrt{\left(\frac{\ell(\ell+1)}{2}\right)}\left[G_{\ell m}^{+}(r)+G_{\ell m}^{-}(r)\right]\right\} Y_{\ell}^{0, m^{\star}}
\end{aligned}
$$

where $N$ is the total number of interfaces. The general expression of the load $p=q_{a}-q_{h}$ is then:

$$
\begin{aligned}
p & =-\rho_{l} U(R+h)+\rho_{l} U(R-w) \\
& -\rho_{c} U(R-w)+\rho_{c} U\left(R-T_{c}-w\right) \\
& \left.-\rho_{m} U\left(R-T_{c}-w\right)+\rho_{m} U\left(R-T_{e}-w\right)\right] \\
& -\rho_{m} U\left(R-T_{e}-w\right)
\end{aligned}
$$

where $\rho_{l}, \rho_{c}$ and $\rho_{m}$ denote respectively the load density, the crustal density and the mantle density. When the load density is equal to the crustal density, this reduces to

$$
p=-\rho_{c} U(R+h)-\left(\rho_{m}-\rho_{c}\right) U\left(R-T_{c}-w\right)
$$

Next, expanding the above potential to first order about $R$ and $R-T_{c}$ yields

$$
p=-\rho_{c}\left(U_{0}+h \frac{d U}{d r}\right)-\left(\rho_{m}-\rho_{c}\right)\left(U_{c}-w \frac{d U}{d r}\right),
$$

where $U_{0}$ and $U_{c}$ are the gravitational potentials at respectively the surface and the base of the crust and $w$ is measured positive downwards. Using $\frac{d U}{d r}=-g_{0}$, where $g_{0}$ is the gravity at surface (assumed positive here and with a same value at the surface and crust-mantle interface), $U_{0} \approx h_{g} g_{0}$ and $U_{c} \approx h_{g_{c}} g_{0}$ (where $h_{g}$ and $h_{g_{c}}$ are the height of a constant potential above the surface and the base of the crust respectively). The load $p$ is then given by

$$
p=g_{0}\left[\rho_{c}\left(h-h_{g}\right)-\Delta \rho\left(h_{g_{c}}-w\right)\right],
$$

where $\Delta \rho=\rho_{m}-\rho_{c}$. This equation is equivalent to that given by Banerdt [1986], noting that he defines $w$ as being positive upwards. If $h_{g_{c}}$ is assumed to be 0 , the expression D4 becomes the relation used in McGovern et al. [2002]

$$
p=g_{0}\left[\rho_{c}\left(h-h_{g}\right)-\Delta \rho w\right] .
$$

If the approximation $h_{g_{c}}=h_{g}$ is assumed, then the relation of Turcotte et al. [1981] is obtained:

$$
p=g_{0}\left[\rho_{c} h-\left(\rho_{m}-\rho_{c}\right) w-\rho_{m} h_{g}\right] .
$$

Acknowledgments. This work was supported by the Programme National de Planétologie and by the the European Commufiity's Improving Human Potential Program under contract RTN2- 2001-00414, MAGE. This is IPGP contribution XX.

\section{References}

\section{Appendix D: Comparison with other methods}

Albert, R. A., R. J. Phillips, A. J. Dombard, and C. D. Brown (2000), A test of the validity of yield strength envelopes with an elastoviscoplastic finite element model, Geophys. J. Int., 140, 399-409.

The expression for the load $p$ given in Turcotte et al. [1981], Banerdt [1986] or McGovern et al. [2002], can be derived from our expressions for $q_{a}$ and $q_{h}$ given by equations 28 and 30 after a series of approximations. We note that in this demonstration, we consider $g_{0}$, the gravity at the surface as a positive value (as used in the articles cited here), and thus the sign convention adopted is $g_{0}=-\frac{d U}{d r}$. First, setting $r^{2}=R^{2}$ and assuming no lateral variation of density, the expression for $q_{a}$ becomes

$$
\begin{aligned}
q_{a} & =-\int_{R-T_{e}-w}^{R+h} \frac{d U}{d r} \rho(r) d r=-\int_{U\left(R-T_{e}-w\right)}^{U(R+h)} \rho(r) d U \\
& =-\sum_{j=1}^{N} \rho(r)\left[U_{j+1}-U_{j}\right],
\end{aligned}
$$

Banerdt, W. (1986), Support of longwavelength loads on Venus and implications for internal structure, J. Geophys. Res., 91, 403-419.

Bertka, C. M., and Y. Fei (1998), Density profile of an SNC model Martian interior and the moment-of-inertia factor of Mars, Earth Planet. Sci. Lett., 157, 79-88.

Brückner, J., G. Dreibus, R. Rieder, and H. Wänke (2003), Refined data of Alpha Proton X-ray Spectrometer analyses of soils and rocks at the Mars Pathfinder site: Implications for surface chemistry, J. Geophys. Res., 108, 8094, doi:10.1029/2003JE002060.

Britt, D. T., and G. J. Consolmagno (2003), Stony meteorite porosities and densities: A review of the data through 2001, Meteorit. Plan. Sci., 38, 1161-1180.

Clévédé, E. and Lognonné P. (2002), Chapter 85.16, Softwares for : Normal modes of the Earth and Planets, Handbook on Earthquake and Engineering Seismology, IASPEI Centennial Publications, H. Kanamori, P. Jennings and W. Lee editors, International Geophysics series, 81B, Academic Press, 2003. 
Comer, R. P., S. C. Solomon, and J. W. Head (1985), Mars: Thickness of the lithosphere from the tectonic response to volcanic loads, Reviews of Geophysics, 23(1), 61-921.

Driscoll, J. R., and D. Healy (1994), Computing fourier transforms and convolutions on the 2-sphere, Adv. appl. Math., 15, $202-250$.

Forsyth, D. W. (1985), Subsurface loading and estimates of flexural rigidity of continental lithosphere, J. Geophys. Res., 90(B14), 12,623-12,632.

Freed, A. M., H. J. Melosh, and S. C. Solomon (2001), Tectonics of mascon loading: Resolution of the strike-slip faulting paradox, J. Geophys. Res., pp. 20,603-20,620.

Frey, H. V., J. H. Roaek, K. M. Shockey, E. L. Frey, and S. E. H. Sakimoto (2002), Ancient lowland on Mars, Geophys. Res. lett., 29(10), 1384, doi:10.1029/2001GL013832.

Hartmann, W. K., and G. Neukum (2001), Cratering chronology and the evolution of Mars, Space Sci. Rev., 96, 165-194.

Head, J. N., H. J. Melosh, and B. A. Ivanov (2002), Martian meteorite launch: High-speed ejecta from small craters, Science, 298, 1752-1756.

Hoogenboom, T., S. E. Smrekar, F. S. Anderson, and G. Houseman (2004), Admittance survey of type 1 coronae on Venus, J. Geophys. Res., E03002, doi:10.1029/2003JE002171.

Jolliff, B. L., J. J. Gillis, L. A. Haskin, R. L. Korotev, and M. A. Wieczorek (2000), Major lunar crustal terranes: Surface expressions and crust-mantle origins, J. Geophys. Res., pp. 41974216.

Jordan, T. H. (1979), Mineralogies, densities and seismic velocities of garnet lherzolites and their geophysical implications, In Boyd, F. R. and Meyer, H. O. A., eds., The mantle sample: Inclusions in kimberlites and other volcanics; Proceedings of the Second international Kimberlite Conference, Volume 2, Washington D. C., Am. Geophys. Union, p. 1-14.

Kiefer, W. S. (2003), Melt in the martian mantle: Shergottite formation and implications for present-day mantle convection on Mars, Meteorit. Planet. Sci., 38, 1815-1832.

Kraus, H. (1967), Thin elastic shells: An introduction to the theoretical foundations and the analysis of their static and $d y$ namic behavior, New York, John Willey, 467 pp.

Lawrence, K. P., and R. J. Phillips (2003), Gravity/Topography admittance inversion on Venus using niching genetic algorithms, Geophys. Res. Lett., $30(19)$, 1994, doi: 10.1029/2003GL017515.

Lemoine, F. G., D. E. Smith, D. D. Rowlands, M. T. Zuber, G. A Neumann, D. S. Chinn, and D. E. Pavlis (2001), An improved solution of the gravity field of Mars (GMM-2B) from Mars Global Surveyor, J. Geophys. Res., 106 (E10), 23,359-23,376.

Lognonné, P., and B. Romanowicz (1990), Modeling of coupled normal modes of the Earth: the spectral method, Geophys. J. Int, 102, 365-395.

Lognonné, P., D. Giardini, B. Banerdt, J. Gagnepain-Beyneix, A. Mocquet, T. Spohn, J. F. Karczewski, P. Schibler, S. Cacho, W. T. Pike, C. Cavoit, A. Desautez, M. Favède, T. Gabsi, L. Simoulin, N. Striebig, M. Campillo, A. Deschamp, J. Hinderer, J. J. Lévéque, J. P. Montagner, L. Rivéra, W. Benz, D. Breuer, P. Defraigne, V. Dehant, A. Fujimura, H. Mizutani, and J. Oberst (2000), The NetLander very broad band seismometer, Planet. Space Sci., 48, 1289-1302.

Lowry, A. R., and S. Zhong (2003), Surface versus internal loading of the Tharsis rise, Mars, J. Geophys. Res., 108(E9), 5099, doi:10.1029/2003JE002111.

McGovern, P. J., S. C. Solomon, D. E. Smith, M. T. Zuber, M. Simons, M. A. Wieczorek, R. J. Phillips, G. A. Neumann, O. Aharonson, and J. W. Head (2002), Localized gravity/topography admittance and correlation spectra on Mars: Implication for regional and global evolution, J. Geophys. Res., 107(E12), 5136, doi:10.1029/2002JE001854.

McGovern, P. J., S. C. Solomon, D. E. Smith, M. T. Zuber, M. Simons, M. A. Wieczorek, R. J. Phillips, G. A. Neumann, O. Aharonson, and J. W. Head (2004), Correction to "Localized gravity/topography admittance and correlation spectra on Mars: Implication for regional and global evolution", $J$. Geophys. Res., E07007, doi:10.1029/2004JE002286.

McKenzie, D., D. N. Barnett, and D.-N. Yuan (2002), The relationship between Martian gravity and topography, Earth Planet. Sci. Lett., 195, 1-16.

McNutt, M. K. (1984), Lithospheric flexure and thermal anomalies, J. Geophys. Res., 89, 11,180-11,194.
McSween, H. Y. (1985), SNC meteorites: Clues to Martian petrologic evolution? Rev. Geophys., 23, 391-416.

Moore, J. G. (2001), Density of basalt core from Hilo drill hole, Hawaii, J. Volcanol. Geoth. Res., 112, 221-230.

Neumann, G. A., M. T. Zuber, M. A. Wieczorek, P. J. McGovern, F. G. Lemoine, and D. E. Smith (2004), The crustal structure of Mars from gravity and topography, J. Geophys. Res., 109, E08002, doi:1029/2004JE002262.

Nimmo, F. (2002), Admittance estimates of mean crustal thickness and density at the Martian hemispheric dichotomy, $J$ Geophys. Res., 107(E11), 5117, doi:10.1029/2000JE001488.

Nyquist, L. E., D. D. Bogard, C.-Y. Shih, A. Greshake, D. Stöffler, and O. Eugster (2001), Ages and Geologic Histories of Martian Meteorites, Space Sci. Rev., 96, 105-164.

Oxburgh, E. R., and E. M. Parmentier (1977), Compositional and density stratification in oceanic lithosphere-causes and consequences, J. Geol. Soc. Lond., 133, 343-355.

Pérez-Gussinyé, and M., Lowry, and A. R., Watts, and A. B., and Velicogna, I. (2004), On the recovery of effective elastic thickness using spectral methods: Examples from synthetic data and from the Fennoscandian Shield, J. Geophys. Res., 109, B10409, doi:10.1029/2003JB002788.

Phillips, R. J., M. T. Zuber, S. C. Solomon, M. P. Golombek, B. M. Jakosky, W. B. Banerdt, D. E. Smith, R. M. E. Williams, B. M. Hynek, O. Aharonson, and S. A. Hauck II (2001), Ancient geodynamics and global-scale hydrology on Mars, Science, 291, 2587-2591.

Phinney, R. A. and R. Burridge (1973), Representation of the elastic-gravitational excitation of a spherical Earth model by generalized spherical harmonics, Geophys. J. R. Astr. Soc., $34,451-487$

Press, W. H., S. A. Teukolsky, W. T. Vetterling, and B. P. Flannery (1992), Numerical recipes in FORTRAN. The art of scientific computing, Cambridge: University Press, 2nd ed, pp. 654 .

Simons, M., S. C. Solomon, and B. H. Hager (1997), Localization of gravity and topography: constraints on tectonic and mantle dynamics of Venus, Geophys. J. Int., 131, 24-44.

Smith, M. D., M. Zuber, H. Frey, J. Garvin, J. Head, D. Muhleman, G. Pettengill, R. Phillips, S. Solomon, H. Zwally, W.B.Banerdt, T. Duxbury, M. Golombek, F. Lemoine, G. Neumann, D. Rowlands, O. Aharonson, P. Ford, A. Ivanov, P. McGovern, J.B. Abshire, R. Afzal, and X. Sun (2001), Mars Orbiter Laser Altimeter MOLA: Experiment summary after the first year of global mapping of Mars, J. Geophys. Res., 106, 23,723-23,735.

Smrekar, S. E., R. Comstock, and F. S. Anderson (2003), A gravity survey of type 2 coronae on venus, J. Geophys. Res., 5090, doi:10.1029/2002JE001935.

Sneeuw, N. (1994), Global spherical harmonic analysis by least squares and quadrature methods in historical perspective, Geophys. J. Int., 118.

Sohl, F., and T. Spohn (1997), The interior structure of mars: Implications from SNC meteorites, J. Geophys. Res., 102, 16131636 .

Solomon, S. C., and J. W. Head (1990), Heterogeneities in the thickness of the elastic lithosphere of Mars: Constraints on heat flow and internal dynamics, J. Geophys. Res., 95, 11,07311,083

Tarantola, A. (1987), Inverse problem theory. Methods for data fitting and model parameter estimation, Elsevier, Amsterdam, 1987, pp 1-88.

Thurber, C. H., and M. N. Toksoz (1978), Martian lithospheric thickness from elastic flexure theory, Geophys. Res. Lett., 5, 977-980.

Turcotte, D. L., R. J. Willemann, W. F. Haxby, and J. Norberry (1981), Role of membrane stresses in the support of planetary topography, J. Geophys. Res., 86(B5), 3951-3959.

Wieczorek, M. A., and R. J. Phillips (1998), Potential anomalies on a sphere: Application to the thickness of the lunar crust, J. Geophys. Res., 103, 1715-1724.

Wieczorek, M. A., and M. T. Zuber (2004), The thickness of the martian crust: Improved constraints from geoid-topography ratios, J. Geophys. Res., 109, 1009-1024.

Wieczorek, M. A., and F. J. Simons (2004), Localized spectral analysis on a sphere, Geophys. J. Int., submitt.

Willemann, R. J., and D. L. Turcotte (1981), Support of topographic and other loads on the moon and on the terrestrial planets, Proc. Lunar Planet. Sci. Conf., 12th, 837-851. 
Yuan, D., Sjogren, W. L., Konopliv, A. S. Kucinskas, A. B. (2001), Gravity field of Mars: A 75th degree and order model, J. Geophys. Res., 106, 23377-23402.

Zhong, S., and M. T. Zuber (2000), Long-wavelength topographic relaxation for self-gravitating planets and implications for the time-dependent compensation of surface topography., J. Geophys. Res., 105(E2), 4153-4164.

Zuber, M. T., and D. E. Smith (1997), Mars without Tharsis, J. Geophys. Res., 102(E12), 28,673-28,685.

Zuber, M. T., S. C. Solomon, R. J. Phillips, D. E. Smith, G. L. Tyler, O. Aharonson, G. Balmino, W. B. Banerdt, J. W. Head, C. L. Johnson, F. G. Lemoine, P. J. McGovern, G. A. Neu- mann, D. D. Rowlands, and S. Zhong (2000), Internal structure and early thermal evolution of Mars from Mars Global Surveyor topography and gravity, Science, 287, 1788-1793.

V. Belleguic, Deutsches Zentrum für Luft- und Raumfahrt (DLR), Institut für Planetenforschung, Rutherfordstrasse 2, 12489 Berlin, Germany (email:Virginie.Belleguic@dlr.de)

P. Lognonné, M. Wieczorek, Département de Géophysique Spatiale et Planétaire, Institut de Physique du Globe de Paris, 4 avenue de Neptune, 94107 Saint-Maur Cedex, France. (email:lognonne@ipgp.jussieu.fr; wieczor@ipgp.jussieu.fr) 